ARTICLE

\title{
Evolution of combinatorial diversity in trans- acyltransferase polyketide synthase assembly lines across bacteria
}

Eric J. N. Helfrich (1) 1,2,6, Reiko Ueoka (10 1,6, Marc G. Chevrette (1) 3,6, Franziska Hemmerling1, Xiaowen Lu4, Stefan Leopold-Messer (10 1, Hannah A. Minas (10 ${ }^{1}$, Adrien Y. Burch ${ }^{5}$, Steven E. Lindow ${ }^{5}$, Jörn Piel ${ }^{1 凶}$ \& Marnix H. Medema (iD ${ }^{4 凶}$

Trans-acyltransferase polyketide synthases (trans-AT PKSs) are bacterial multimodular enzymes that biosynthesize diverse pharmaceutically and ecologically important polyketides. A notable feature of this natural product class is the existence of chemical hybrids that combine core moieties from different polyketide structures. To understand the prevalence, biosynthetic basis, and evolutionary patterns of this phenomenon, we developed transPACT, a phylogenomic algorithm to automate global classification of trans-AT PKS modules across bacteria and applied it to 1782 trans-AT PKS gene clusters. These analyses reveal widespread exchange patterns suggesting recombination of extended PKS module series as an important mechanism for metabolic diversification in this natural product class. For three plantassociated bacteria, i.e., the root colonizer Gynuella sunshinyii and the pathogens Xanthomonas cannabis and Pseudomonas syringae, we demonstrate the utility of this computational approach for uncovering cryptic relationships between polyketides, accelerating polyketide mining from fragmented genome sequences, and discovering polyketide variants with conserved moieties of interest. As natural combinatorial hybrids are rare among the more commonly studied cisAT PKSs, this study paves the way towards evolutionarily informed, rational PKS engineering to produce chimeric trans-AT PKS-derived polyketides.

\footnotetext{
${ }^{1}$ Institute of Microbiology, Eidgenössische Technische Hochschule (ETH) Zürich, Zürich, Switzerland. ${ }^{2}$ Institute for Molecular Bio Science, Goethe University Frankfurt, Frankfurt am Main, Germany. ${ }^{3}$ Wisconsin Institute for Discovery, Department of Plant Pathology, University of Wisconsin-Madison, Madison, WI, USA. ${ }^{4}$ Bioinformatics Group, Wageningen University, Wageningen, The Netherlands. ${ }^{5}$ Department of Plant and Microbial Biology, University of California at Berkeley, Berkeley, CA, USA. ${ }^{6}$ These authors contributed equally: Eric J. N. Helfrich, Reiko Ueoka, Marc G. Chevrette. ${ }^{凶}$ email: jpiel@ethz.ch; marnix.medema@wur.nl
} 


\section{T} rans-acyltransferase polyketide synthases (trans-AT PKSs) are giant bacterial enzymes that assemble highly diverse and complex polyketide natural products. Some of these, such as mupirocin and virginiamycin, are used as antibiotics in human and veterinary medicine $e^{1,2}$, and many others show promising drug-like bioactivities ${ }^{3-6}$. In addition, trans-AT PKSderived polyketides are of ecological relevance as they have been linked to symbiotic interactions ${ }^{7-9}$ and pathogenicity ${ }^{10-12}$. TransAT PKSs are broadly distributed across the bacterial tree of life, including many chemically underexplored lineages ${ }^{13-15}$. Like the well-studied cis-AT PKSs, they form multifunctional assembly lines composed of multiple enzymatic modules, each of which is responsible for the incorporation and facultative processing of an acyl-CoA-derived building block. In the trans-AT systems, these units are usually malonyl residues that are attached by one or few free-standing acyltransferase (AT) enzymes to an acyl carrier protein (ACP) domain present in each module. A ketosynthase (KS) domain in the downstream module then uses this building block to elongate the presented intermediate, resulting in a $\beta$-keto thioester. Additional optional domains, either located within a module or also acting in trans, can further modify this thioester in the $\alpha$-to- $\gamma$ region to generate a product that is passed on to the next module. When the polyketide backbone is fully assembled, it is released from the PKS, typically by a thioesterase (TE) domain. Additional post-PKS enzymes may modify the polyketide backbone to produce the mature polyketide ${ }^{13,16}$. The diversity of module architectures $(>150 \text { known variants })^{8}$ and enzymatic domains found in trans-AT PKSs is remarkable, and far exceeds the architectural diversity of cis-AT PKSs ${ }^{13,17}$. In many cases, PKSs form hybrids with nonribosomal peptide synthetases (NRPSs) to produce hybrid polyketide-peptide backbones ${ }^{13}$.

Phylogenetic studies suggest that trans-AT PKSs evolve in a distinct fashion compared to textbook cis-AT PKSs. In cis-AT PKS systems, in which an AT domain is present in every module, pathways appear to largely arise from module duplication and subsequent divergence ${ }^{18,19}$, probably in combination with gene conversion ${ }^{20}$. This evolutionary scenario is reflected in the phylogeny of KS domains, which are usually monophyletic within the same cis-AT PKS family ${ }^{21}$. In contrast, the phylogeny of KS domains from trans-AT PKSs tightly correlates with the $\alpha$-to- $\gamma$ structural region of their thioester substrates ${ }^{17,22,23}$, which is at least in part a consequence of their substrate selectivity ${ }^{22,24}$. Accordingly, KS domains in the same assembly line that do not recognize the same substrate type are usually more remotely related and do not group together in phylogenetic trees. This correspondence between KS sequences and the structure of their accepted polyketide intermediates, referred to as the trans-AT PKS correlation rule ${ }^{17}$, permits detailed predictions of polyketide structures from trans-AT PKS biosynthetic gene clusters (BGCs) ${ }^{17,22,23,25-30}$.

Previous studies suggested for pederin-type compounds ${ }^{31}$ and a family of actin-binding macrolides ${ }^{29}$, respectively, that natural product structures within each of these compound groups have diversified by horizontal gene transfer and combinatorial reorganization of large trans-AT PKS gene fragments encompassing multiple PKS modules, here termed module blocks. At the chemical level, this mechanism creates complex chimeric polyketide families that share extended structural moieties, reminiscent of compounds generated by mix-and-match combinatorial chemistry ${ }^{29,31}$. In contrast, such hybrid assembly lines and their associated chimeric metabolites are not typically observed for textbook cis-AT PKSs ${ }^{23}$. In actin-binding macrolides, PKS chimerization enables extensive structural diversification, as well as preservation of pharmacophore moieties in otherwise structurally unrelated compounds.

We wanted to explore whether the ability to form hybrid assembly lines is a general feature of trans-AT PKSs. If so, this property might enable an evolution-guided genome mining strategy to discover polyketides with conserved substructures. Moreover, combinatorial plasticity would constitute a desirable property for the biosynthetic engineering of trans-AT PKS megasynthases. Hence, lessons learned from natural recombination and pathway evolution could inform how PKS fragments can be successfully recombined to generate nonnatural PKS assembly lines.

In this work, we present the trans-AT PKS Annotation and Comparison Tool (transPACT), an algorithm that automatically assigns trans-AT PKS-derived KS domains to functional clades and identifies contiguous series of PKS modules, i.e., module blocks, shared between different PKS assembly lines. We describe the application of transPACT in a global analysis of 1782 transAT PKS BGCs in publicly available genomes, and show how combinatorial novelty evolved across a wide range of trans-AT PKS families from different phyla. Our data reveal cryptic evolutionary relationships between seemingly non-related pathways and demonstrate that, in contrast to cis-AT PKS systems, combinatorial assembly is a widespread and general phenomenon in trans-AT PKSs. We apply these insights to understand natural product biosynthesis in three plant-associated bacteria and show the utility of transPACT to uncover cryptic evolutionary relationships between distinct polyketide families and to conduct targeted, moiety-based polyketide mining even from fragmented draft genome assemblies. This study provides evidence for the origin of chemical innovation in trans-AT PKSs and paves the way toward rational, evolution-inspired engineering of nonnatural hybrid PKSs.

\section{Results}

Identification of conserved module blocks in annotated BGCs using transPACT. To identify signatures of mosaic-like evolution across trans-AT PKSs, we developed the computational tool transPACT (Fig. 1). As input for a search algorithm, we generated a maximum-likelihood phylogenetic tree of $647 \mathrm{KS}$ domains (SI Fig. 1) from trans-AT PKSs with known products, in which isofunctional clades consisting of KS sequences with shared substrate types (for example, KSs accepting thioesters with $\beta$-hydroxyl groups) were manually annotated based on knowledge on the biosynthetic pathways or proposed biosynthetic models ${ }^{13,16}$. transPACT then uses this tree as a reference for pplacer, a maximum-likelihood phylogenetic placement algorithm ${ }^{32,33}$, to assign query sequences to functional clades. Benchmarking on a set of 12 recently published trans-AT PKS assembly lines that were not part of the training set showed an accuracy of $81.2 \%$, with regard to the chemistry associated with these functional clades (SI Dataset 1), which is on par with the results of the state-of-the-art algorithm for trans-AT PKS-derived polyketide structure prediction TransATor ${ }^{17}(82.5 \%)$. Thus, transPACT annotates PKS assembly lines with functional clade assignments for each module, which can be used to identify blocks of KS types that recur across different PKSs.

To test the capability of transPACT to identify module blocks that reflect shared chemical substructures in corresponding polyketide products, we initially used all 49 characterized transAT PKSs reported by Helfrich and Piel ${ }^{13}$ that were included in the reference tree. We found that 46 (94\%) out of the 49 known trans-AT PKSs shared at least one contiguous pair of modules with another assembly line, and $34(69 \%)$ shared at least two pairs; network analysis based on these data led to the identification of patterns of module sharing between various families of PKSs (Fig. 2a). Analysis of the polyketide structures confirmed that PKSs with shared module blocks generate natural products with common substructures (SI Fig. 2 and SI Table 1). 


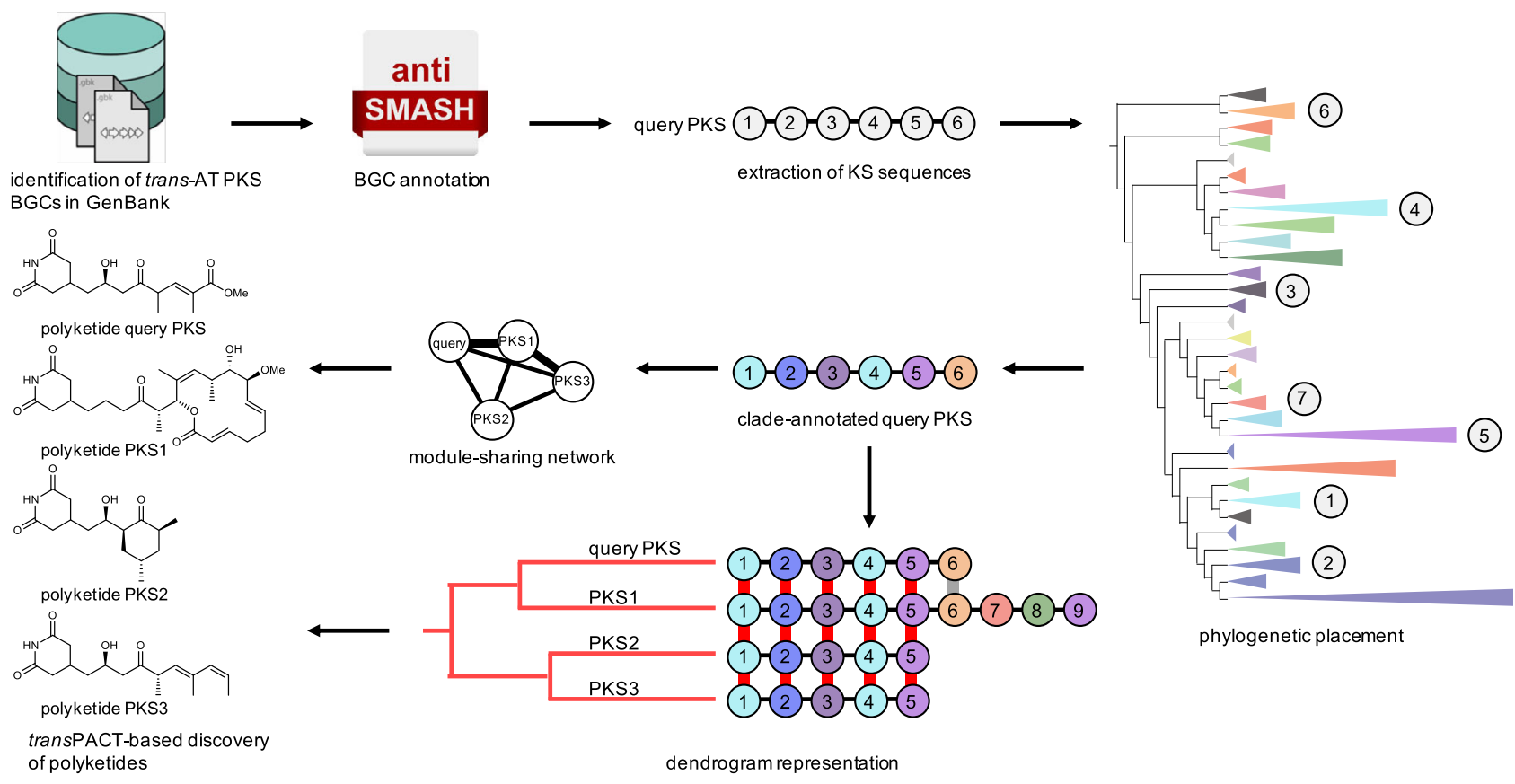

Fig. 1 transPACT Workflow. One thousand seven hundred eighty two trans-AT PKS BGCs were retrieved from GenBank and subjected to antiSMASH analysis ${ }^{38}$. Trans-AT PKS KS sequences were extracted, followed by phylogenetic placement to annotate query KS sequences that correlate with substrate specificity. Module sharing networks and dendrogram representations were computed and used for, e.g., subsequent genome mining studies resulting in the isolation of novel members of trans-AT PKS families, the identification of novel trans-AT PKS scaffolds, and culturable representatives of uncultured producers of polyketides and evolutionary insights.

Further validating the transPACT analysis, one of the familial groupings within the network contains the three PKSs for the combinatorial actin-binding macrolides tolytoxin (1), luminaolide (2), and misakinolide (3) (Fig. 2a, b) ${ }^{29}$. Indeed, the detected conserved motifs are in perfect agreement with our previous study showing that large parts of the BGCs have recombined to generate these macrolides ${ }^{29}$. In addition to these reported observations, this analysis also showed that the predicted substrates of KSs present in modules 3 and 4, as well as 6-17 are surprisingly conserved among all three PKSs, indicating that, in the misakinolide PKS, modules 1 and 2, as well as 5 might have been exchanged with other modules during evolution, while modules 3 and 4 , as well as $6-17$, were retained (Fig. 2b, and SI Figs. 1 and 2).

The BGCs associated with the production of pederin-like compounds are believed to have arisen from a remarkably extensive inter-phylum gene transfer and divergent evolution to yield pederin (4) (Gammaproteobacteria $)^{34}$, nosperin (7) (Cyanobacteria) ${ }^{26}$, onnamide (6) ("Tectomicrobia") 35,36 , diaphorin (5) (Betaproteo-

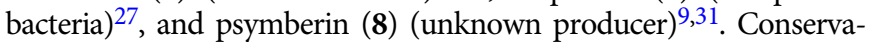
tion of module sharing across the pederin-type PKSs indicated three distinct structural motifs, which are reflected in the PKS module architecture (Fig. 2c and SI Fig. 2). The first five modules of the pederin-like PKS family are highly conserved with the exception of the psymberin PKS, which lacks the second module. This conservation on the PKS level is reflected in the highly similar Western parts of pederin-like compounds. The four modules following the first conserved module block in the onnamide PKS have either been excised in the nosperin PKS or have been inserted into a nosperin-like precursor PKS to form the onnamide PKS. In addition, two of the terminal three modules of the onnamide PKS likely fused to the $\mathrm{N}$-terminal part of the nosperin PKS or vice versa. Pederin (4) and diaphorin (5) are likely biosynthesized via oxygen insertion into the polyketide backbone ${ }^{27,34}$, followed by the hydrolysis of the resulting ester ${ }^{37}$. At the PKS level, a module block stretching the two modules 10 and 11 is present in the pederin and diaphorin PKSs for this oxidative modification ${ }^{37}$ and a subsequent elongation. In the case of the pederin PKS, this bimodule is neighbored by the large module block that is conserved between all pederin-like PKSs on the upstream side and the downstream block of the onnamide PKS that is shared between the pederin, nosperin and onnamide PKSs. The C-terminal modules of the psymberin PKS, however, do not contain KSs shared with any other PKS in this family, suggesting a different origin. The verification of the previously reported evolutionary relationship of the actin inhibitor and pederin-type PKSs by our algorithm indicated that transPACT can detect as-yet undetected combinatorial recombination events in other assembly lines. Moreover, transPACT uncovered cryptic evolutionary relationships between the calyculin and rhizoxin PKSs (Fig. 2 and SI Fig. 2), particularly in a stretch of conserved modules responsible for the biosynthesis of the polyene region of both associated polyketides. Similar cryptic evolutionary relationships were observed between the bacillaene and thailandamide PKSs (likewise the polyene section; Fig. 2 and SI Fig. 2), indicating that transPACT is able to identify previously undiscovered evolutionary relationships even in well-characterized PKSs.

Widespread transfer of module blocks across trans-AT PKSs. To identify combinatorial patterns at a global scale, we compiled all 1782 trans-AT PKS assembly lines from genomes publicly available in GenBank (based on antiSMASH 3.0 predictions ${ }^{38}$ ) and used transPACT to interrogate trans-AT PKS evolution across the whole enzyme class (Fig. 1). We first carried out a multistep quality control on the predicted BGCs to make sure that (i) the BGCs contained at least two KS domains (in total, 1509 out of 1782 BGCs were selected for further analysis), and (ii) for each BGC, we excluded cis-AT PKS modules containing a KS and an AT domain. For all assembly lines and KS domains that passed these criteria, the pplacer maximum-likelihood phylogenetic 
a

b

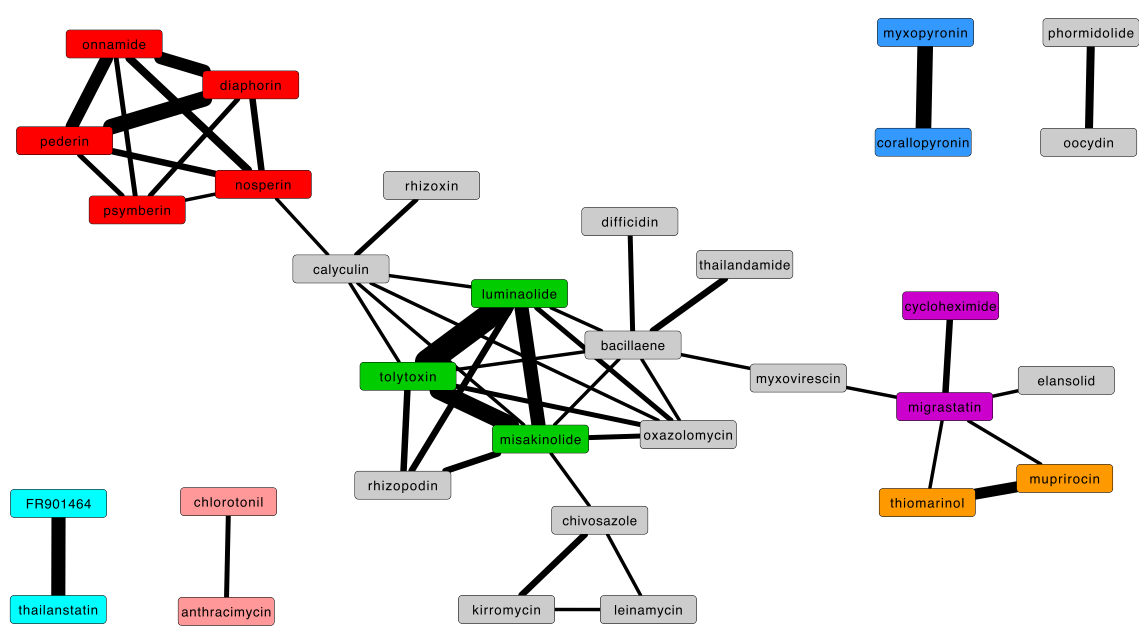

tolytoxin (1) PKS

(Scytonema sp. PCC 10023)

luminaolide (2)PKS

(Planktothrix paucivesiculata PCC 9631

misakinolide (3) PKS

(Candidatus Entotheonella serta)
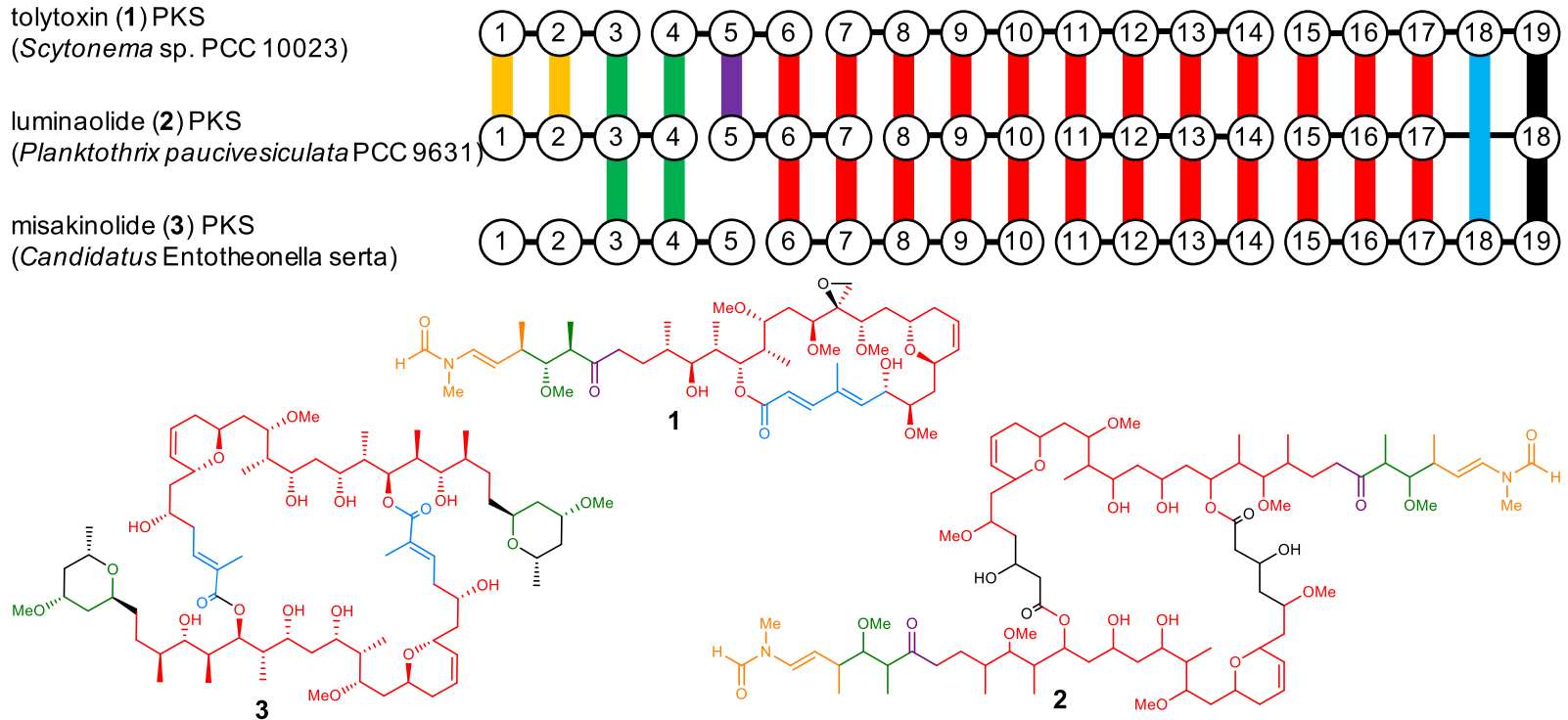

c

pederin (4)PKS

(Pseudomonas spp.)

diaphorin (5) PKS

(Candidatus Profftella armatura)

onnamide (6) PKS

(Candidatus Entotheonella factor)

nosperin (7) PKS

(Nostoc sp.)

psymberin (8) PKS

(producerunknown)
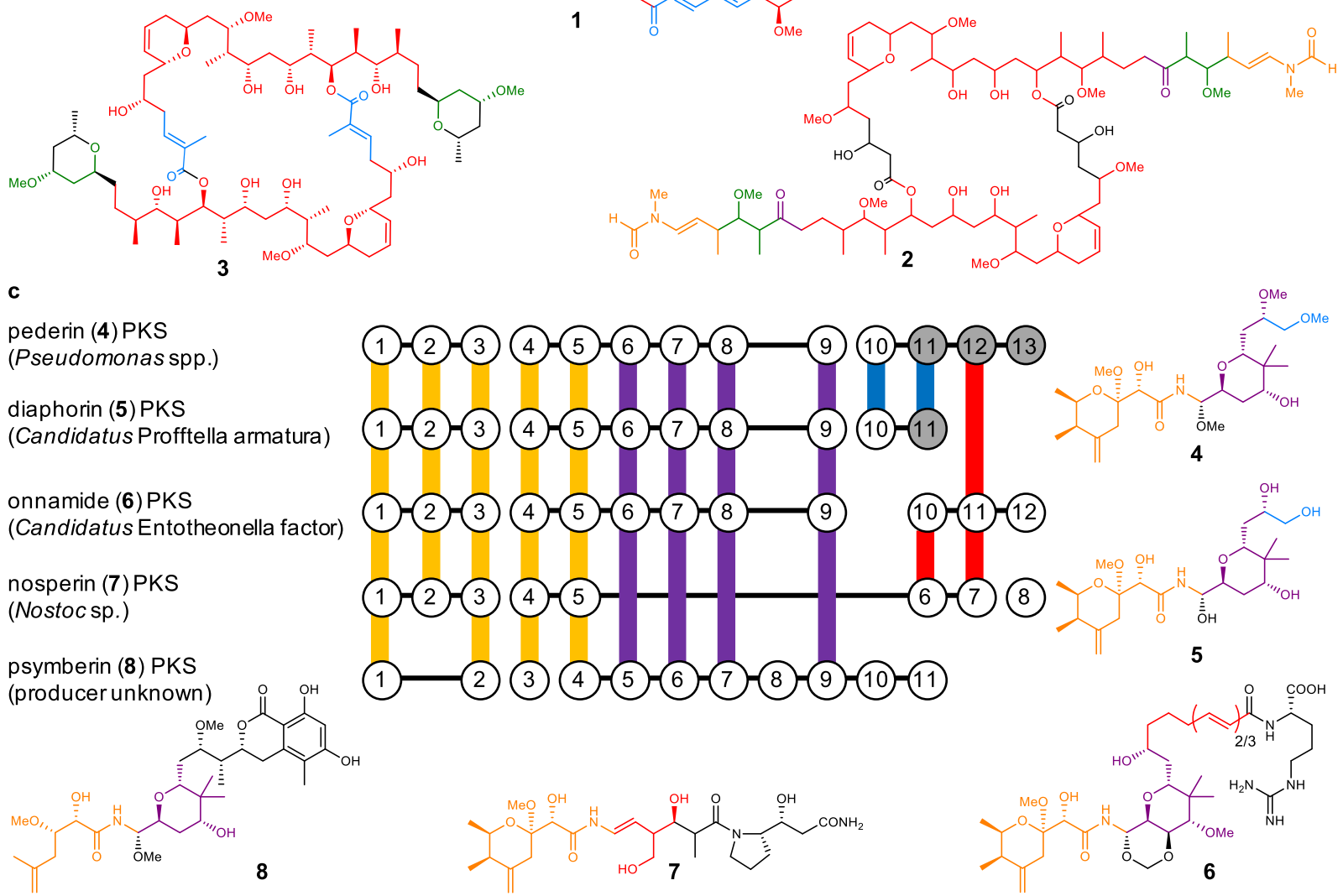

) 
Fig. 2 Conserved PKS module blocks ( $\geq 2$ conserved consecutive modules) identified across all characterized trans-AT PKSs and within the misakinolide, and pederin-type PKS families. a PKS network of all characterized PKSs that share motifs with other characterized PKSs. The edge line width indicates the number of shared modules. PKS families are color-coded as follows: red: pederin-like PKSs, green: misakinolide-like PKSs, light blue: spliceostatin PKSs, violet: glutarimide PKSs, orange: mupirocin/thiomarinol PKSs, blue: corallopyronin/myxopyronin PKSs, rose: chlorotonil/anthracimycin PKSs, and gray: PKSs that do not belong to a characterized trans-AT PKS subfamily. b Evolutionary relationship between misakinolide-like PKSs. Conserved PKS module blocks and the corresponding structural moieties of polyketides are shown in the same colors. Vertical lines represent KSs that cluster together, horizontal lines connect domains of proteins. c Evolutionary relationship between pederin-type PKSs. Gray circles indicate KSs downstream of putative oxygen insertion events. Oxygen insertion, followed by hydrolysis of the formed ester results in a shortened polyketide. An editable Cytoscape network file is available as source data.

a
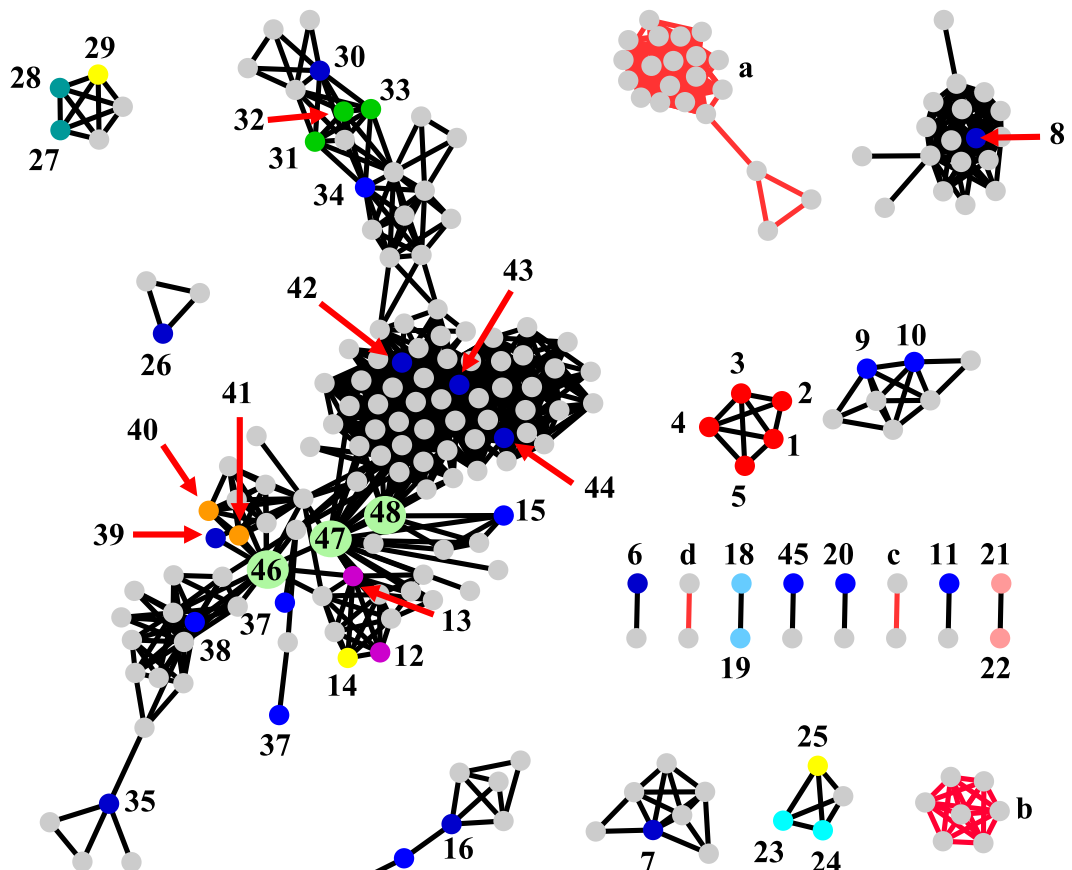

37
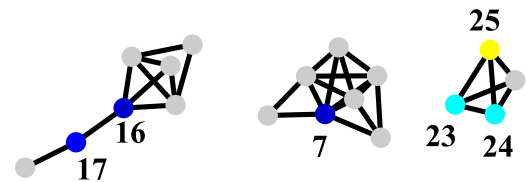

b

\begin{tabular}{|lcccc|}
\hline & 2 modules & 3 modules & 4 modules & $\geq 5$ modules \\
\hline characterized trans-AT PKSs architectures & 28 & 12 & 9 & 16 \\
\hline unique trans-AT PKSs architectures & 963 & 698 & 220 & 437 \\
\hline
\end{tabular}

Fig. 3 Conserved PKS motifs across all 1782 trans-AT PKS BGCs analyzed in this study. a Network representation ( $\geq 3$ conserved consecutive modules). Individual PKSs are represented as nodes and the interconnecting edges indicate module pairs shared by two PKSs. 1: diaphorin; 2: nosperin; 3: onnamide; 4: pederin; 5: psymberin; 6: griseoviridin/virginiamycin, 7: leinamycin; 8: macrolactin; 9: kirromycin; 10: chivosazole; 11: basiliskamide; 12: 9-methylstreptimidone/cycloheximide; 13: migrastatin/dorrigocin; 14: secimide; 15: myxovirescin; 16: rhizoxin; 17: calyculin; 18: myxopyronin; 19: corallopyronin; 20: bryostatin; 21: chlorotonil; 22: anthracimycin; 23: FR901464; 24: thailanstatin; 25: spliceostatin; 26: enacyloxin; 27: oocydin; 28: phormidolide; 29: gynuellalide; 30: rhizopodin; 31: luminaolide; 32: misakinolide; 33: tolytoxin; 34: oxazolomycin; 35: etnangien; 36: batumin/ kalimantacin; 37: sorangicin; 38: SIA7248; 39: elansolid; 40: thiomarinol; 41: mupirocin; 42: thailandamide; 43: bacillaene; 44: difficidin; 45: patellazole; 46: P. durus|CP009288|c3; 47: B. gladioli|CP009322|c8; 48: G. sunshinyii YC6258|CP007142|lacunalide. Colored nodes: characterized PKSs as depicted in Fig. 2a; gray: orphan PKSs; yellow nodes: PKSs for which the corresponding polyketide was identified in this study; light green nodes: nodes with a high transitivity (i.e., connected to several other nodes representing different PKS families); red edges: novel module blocks conserved in biosynthetic pathways. a: mainly Burholderia spp.; b: mainly Paenibacillus spp.; c: Candidatus Magnetomorum; d: Pseudomonas spp. Note: individual nodes represent unique trans-AT PKS architectures, can be composed of multiple GenBank files and might be responsible for the biosynthesis of distinct polyketides (e.g., cycloheximide and 9-methylstreptimidone). b Number of shared modules between all characterized PKSs and all 1782 trans-AT PKS BGCs analyzed in this study. An editable Cytoscape network file is available as source data.

We then merged this dataset with the reference data and made the dataset nonredundant (removing duplicates of assembly lines that contain KSs grouping with the same clades in the same order). A network visualization of conserved module pair sharing between BGCs (Fig. 3) showed that multiple module blocks are shared by otherwise unrelated assembly lines, indicating widespread evolutionary modularity of trans-AT PKSs arising from recombination. We also visualized the architectural relationships in a dendrogram (Fig. 4 and SI Dataset 2), using a distance based on a combination of the Jaccard index of shared KS clades, their copy number differences, and synteny conservation. The colorcoding in the dendrogram, which corresponds to the succession of KS types and thus to moieties present in a polyketide, reveals architectural and evolutive patterns across the bacterial kingdom. 


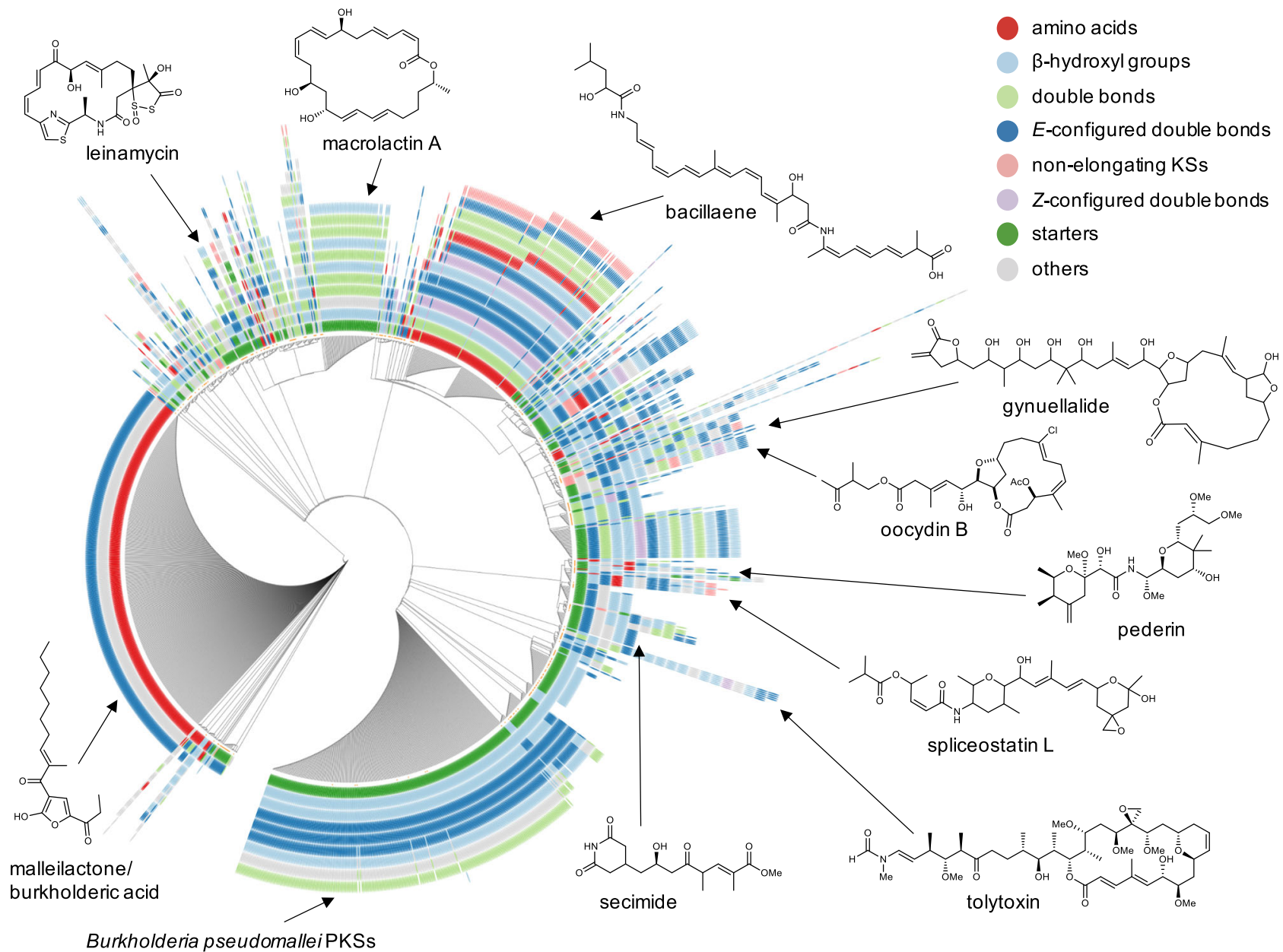

Fig. 4 Dendrogram representation of conserved PKS motifs across all 1782 trans-AT PKSs analyzed in this study. Colors represent classes of KS clades that correlate with polyketide motifs. Selected polyketides are shown that are either associated with PKSs characterized in this study or belong to large PKS families. An interactive representation of the dendrogram can be accessed here: https://itol.embl.de/tree/474115015487031585082885\#.

Together, the network and dendrogram permit rapid exploration of trans-AT PKS biosynthetic diversity by accelerating the detection of novel assembly line variants, conserved polyketide motifs, groups of bacteria harboring particularly diverse PKSs, and chemically neglected taxa.

The network analysis identified several patterns that corroborate recent findings in literature, which provided a first confirmation of the correctness of the approach: for example, transPACT identified a significant expansion of the leinamycin family of PKSs with additional variants, which is in agreement with two recent studies describing new members of this PKS family (Fig. 3) ${ }^{39,40}$. Likewise, many other PKS families were expanded by transPACT. In many cases, identical trans-AT PKS architectures, each represented by one node in the network visualization (Fig. 3), were identified that occur in multiple members of the same species, related species, or even different genera, indicating the widespread production of the same polyketide scaffold. These PKSs frequently cluster together with similar PKSs to form families that only differ by gene order or by one or few modules, thus giving rise to putatively highly similar polyketide scaffolds (SI Figs. 3 and 4). Abundant trans-AT PKS families include those responsible for the biosynthesis of (congeners of) the pathogenicity factor mallailactone/burkholderic acid (>300 PKS assembly lines), macrolactin (54 PKS assembly lines), difficidin (62 PKS assembly lines), and bacillaene (134 PKS assembly lines) (SI Fig. 3). Interestingly, most of these PKS families were identified in individual genera with notable exceptions. Difficidin and bacillaene-like PKSs are almost exclusively encoded in Bacillus genomes with the exception of a few Staphylococcus pneumoniae genomes, indicating horizontal gene transfer between different families of Firmicutes. While the Bacillus species harboring the bacillaene or difficidin PKSs are considered plant-protective ${ }^{41}, S$. pneumoniae is a human pathogen. Finally, the analysis suggests that the plant diseases caused by Ralstonia solanacearum, which infects a broad range of different agricultural crops $^{42}$, might involve the same pathogenicity factor as in rice seedling blight, i.e., rhizoxins ${ }^{43}$. In accordance with recent studies, transPACT also identified BGCs that are almost identical to the rhizoxin BGC in the genomes of the human pathogen Chromobacterium violaceum ${ }^{44}$ and Ralstonia syzygii R22945. Common features of PKS familiar groupings include the shortening of PKSs (bacillaene, oocydin, thailandamide, glutarimide, and difficidin-type assembly lines), module duplications, or module swaps as the only differences between otherwise identical PKS architectures (e.g., spliceostatin PKSs; SI Figs. 3 and 4). It should be noted though, that such cases should be treated with caution, as misassemblies cannot be fully ruled out, and the original raw reads for the genomes are frequently not publicly available to confirm the correctness of the assemblies easily.

In addition to permitting the rapid identification of conserved polyketide families, the network and dendrogram visualizations 
suggested extensive transfer of module blocks and formation of chimeric PKSs. For blocks of 2, 3, 4, and 5 or more modules, hundreds of conserved motifs were identified in the 1782 assembly lines within each category (Fig. 3b). Highly conserved module blocks corresponding to polyketide moieties that are particularly common might correlate with evolutionary success, as would be expected for molecule parts imparting bioactivity. One family of trans-AT PKSs, responsible for the biosynthesis of glutarimidecontaining polyketides was greatly expanded in the dendrogram (Fig. 4a and SI Dataset 2) and the network representation (Fig. 3). Glutarimides are a diverse family of $>50$ polyketides that harbor the characteristic glutarimide residue ${ }^{13}$. Many of these, such as cycloheximide ${ }^{46}$, are cytotoxins, and some members (i.e., lactimidomycin, iso-migrastatin, and migrastatin $)^{47}$ show promising anticancer bioactivities and serve as drug leads ${ }^{13,47,48}$. In total, our analysis revealed 43 PKSs or PKS fragments as candidates for the production of glutarimide-containing polyketides. While, to our knowledge, such compounds have almost exclusively been reported from Streptomyces strains, we found matching glutarimide module blocks in various gammaproteobacterial pathogens, including Burkholderia gladioli and Pseudomonas spp. (SI Fig. 3). Further predicted glutarimide PKSs were identified in Magnetospirillum and Geobacter, two genera with poorly understood chemistry (Fig. 4).

We also observed several nodes with a high transitivity (i.e., connected to multiple other network nodes from different PKS families), which suggested the formation of either larger assembly lines from smaller ones or vice versa. Notable examples include the recently characterized lacunalide ${ }^{49}$ PKS in Gynuella sunshinyii YC6258 (CP007142) and the metabolically unassigned cluster 3 in Paenibacillus durus (CP009288) and cluster 8 in B. gladioli (CP009322; Fig. 3, light green nodes).

The network representation also revealed potential outliers, i.e., trans-AT PKS assembly lines with products unrelated to known molecules, and therefore with high potential for the discovery of uncharacterized polyketide scaffolds in future genome mining efforts (Fig. 3, red edges). We identified several familial groupings of PKSs (between 2 and 20 distinct PKSs) not containing any reported representative. These PKS families were predominantly observed in Burkholderia, Paenibacillus, Pseudomonas, Azospirillum, Streptococcus, and Bacillus spp. genomes. It remains to be determined whether these genera are particularly rich sources of undescribed trans-AT PKS-derived polyketide scaffolds, or whether the observed numbers are a result of a bias originating from the overrepresentation of the sequenced genomes in GenBank. The identification of shared module blocks in PKSs encoded in these producers shows that transPACT cannot only identify unknown producers of known polyketides, producers of variants of the same scaffold, and evolutionarily successful substructures, but can also be used to prioritize polyketide scaffolds for future genome mining studies (SI Datset 2 and SI Fig. 3).

transPACT-guided isolation of a new glutarimide-containing polyketide. To test whether our computational approach can lead to functional insights into the chemical diversity of bacteria, we selected a BGC that transPACT predicted to belong to an unreported glutarimide-containing polyketide in the plant pathogen Pseudomonas syringae pv. syringae, which causes leaf spot diseases in various agricultural crops ${ }^{50}$. Like other PKSs responsible for the biosynthesis of glutarimide-containing polyketides, the selected PKS in this bacterium shows a strong conservation in its first five modules (Figs. 4 and 5a, red), with the exception of module 4 which could not be predicted with high confidence by transPACT.
The presence of the glutarimide moiety was corroborated by structural predictions based on KS phylogeny (SI Fig. 4) and TransATor analysis ${ }^{17}$. TransATor is a recently reported genome mining tool that complements transPACT and that can be used for the structural prediction of trans-AT PKS-derived polyketides from PKS sequences. Downstream of the fifth module, the glutarimide-type PKSs (and corresponding metabolites) vary extensively, giving rise to dozens of different glutarimide-containing polyketides, of which cycloheximide $(\mathbf{1 0})^{46}$, 9-methylstreptimidone $(\mathbf{1 1})^{46}$, and migrastatin $(\mathbf{1 2})^{47}$ are representatives (Fig. 5a). Our analysis (Fig. 5a) suggested the conserved PKS module block corresponding to the glutarimide residue in the P. syringae trans-AT PKS BGC (termed sec) is followed by a module that is homologous to migrastatin-like PKSs suggesting that the core structure of the corresponding polyketide likely resembles a truncated version of migrastatin. To test whether the sec PKS is indeed responsible for the production of a glutarimide-containing metabolite, $P$. syringae $\mathrm{pv}$. syringae was cultivated for chemical analysis.

In initial trials, we were unable to identify a candidate for the predicted structure using various media and culturing conditions. We therefore generated a nonproducing mutant by disruption of the PKS gene $\sec D$ (SI Fig. 5 and SI Table 2). Ultrahighperformance liquid chromatography-high-resolution electrospray ionization-mass-spectrometry-based (UHPLC-HRESI-MS) comparative metabolomics of the wild-type (WT) and the knockout strains did not reveal differences under standard culturing conditions. However, changing the medium composition to mimic a natural plant environment by addition of fructose triggered the biosynthesis of a compound uniquely present in the WT extracts (SI Fig. 6). Isolation by repetitive rounds of reverse-phase HPLC and structure elucidation by high-resolution MS, 1D and 2D NMR experiments yielded a new polyketide that we termed secimide (9) (Fig. 5 and SI Figs. 7-12). The absolute configuration at C8 was determined by Mosher's ester analysis (SI Figs. 13-23 and SI Table 3). The core structure of secimide resembles a truncated migrastatin-like or an extended cycloheximide-type polyketide as predicted. Notably, however, it is one elongation $\left(C_{2}\right)$ unit shorter than the prediction, suggesting that either the final module is skipped during biosynthesis (SI Fig. 5, route I), or the polyketide is shortened in a tailoring reaction (SI Fig. 5, route II), perhaps catalyzed by the oxygenase SecA (SI Table 4).

Unraveling evolutionary relationships between phormidolideand oocydin-type polyketides. Another subcluster in the network of shared PKS module blocks (Fig. 2) includes the two architecturally related cyanobacterial PKSs for the biosynthesis of phormidolide (13) (phor PKS) from Leptolyngbya sp. ISBN3Nov-94-8 ${ }^{51}$ and leptolyngbyalide (14) (lept PKS) from Leptolyngbya sp. PCC $7375^{17}$. Surprisingly, the network also indicated the existence of a phormidolide-like PKS in the taxonomically distant halophile G. sunshinyii YC6258, a halophilic Oceanospirillales bacterium associated with Carex plant roots (Fig. 3b, SI Fig. 24 and SI Table 4) ${ }^{49,52}$. Its PKS (termed the gyn PKS) shows a high degree of conservation with the phor and lept PKSs, sharing 14 out of 17 modules. Results obtained from the transPACT analysis were in good agreement with manual analysis by phylogenetic inference (SI Fig. 4) and TransATor analysis ${ }^{17}$.

To identify the suggested gyn product, a $10 \mathrm{~L}$ G. sunshinyii culture was extracted and analyzed. Using the structure obtained from KS substrate predictions (TransATor analysis ${ }^{17}$ ) as guidance, a matching candidate metabolite was identified by UHPLC-HRESI-MS. Its molecular formula $\left(\mathrm{C}_{40} \mathrm{H}_{62} \mathrm{O}_{12}\right)$, as suggested from the exact mass $\left(m / z\right.$ 757.4147 $[\mathrm{M}+\mathrm{Na}]^{+}$, 
a

secimide (9) PKS

(Pseudomonas syringae

pv. syringae)

cycloheximide (10) PKS

(Streptomyces griseus)

9-methylstreptimidone (11) PKS

(Streptomyces himastatinicus)

migrastatin (12) PKS

(Streptomyces platensis)
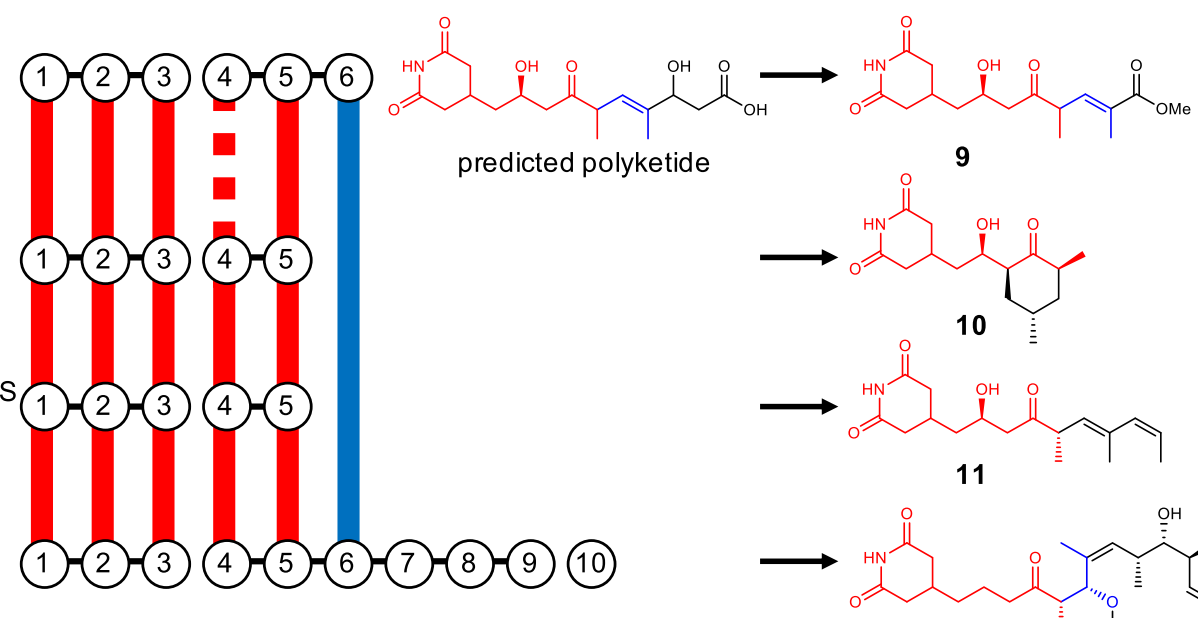

11

b

phormidolide A (13)PKS

(Leptolyngbyasp.)

leptolyngbyalide A (14)PKS

(Leptolyngbyasp.)

gynuellalide (15)PKS

(Gynuella sunshinyii)

oocydin A/B (16/17) PKS

(Serratia plymuthica)

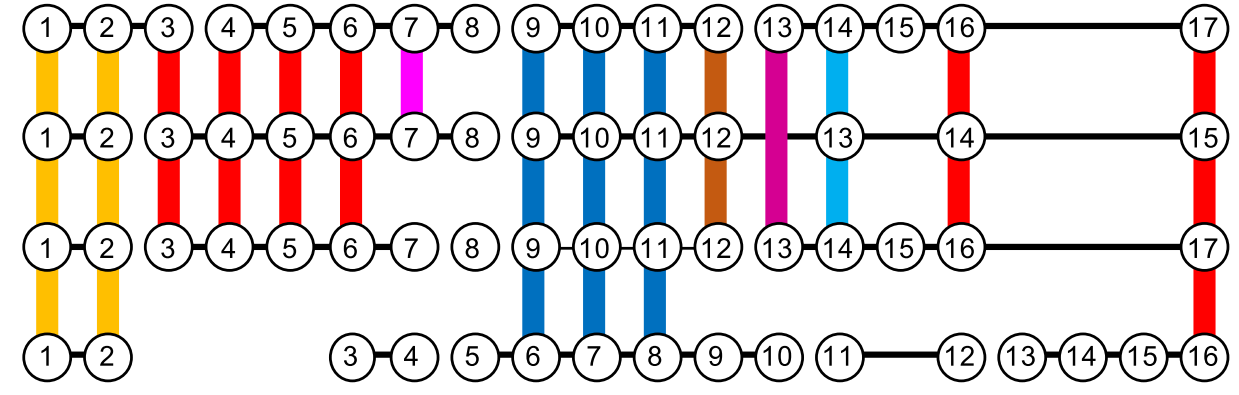

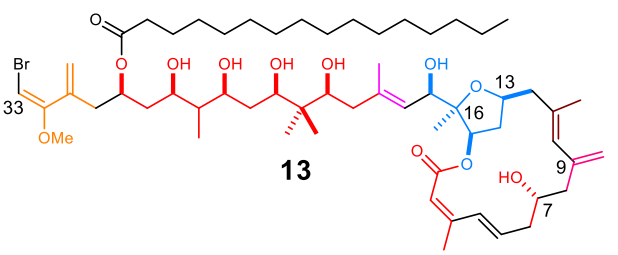

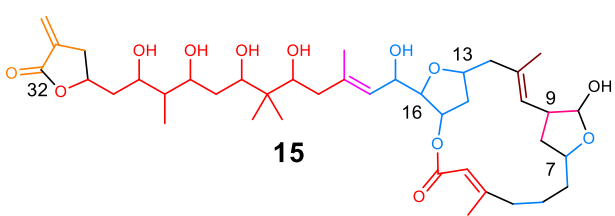

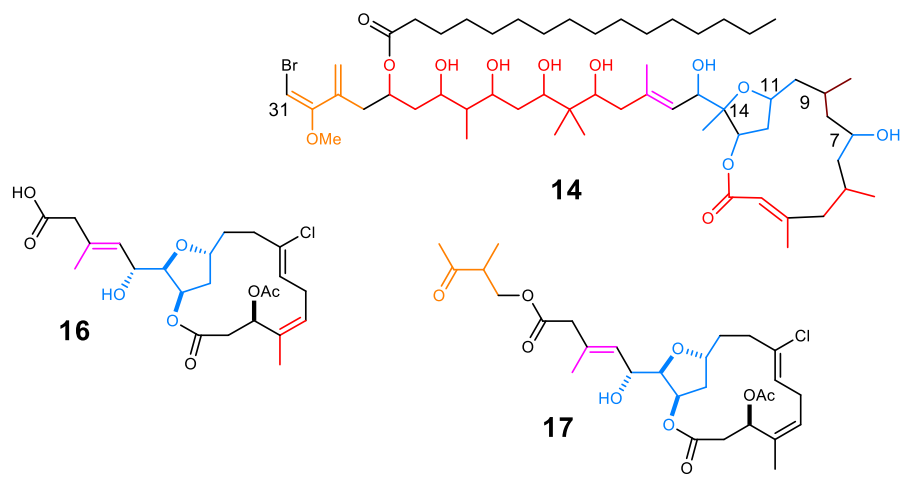

thailanstatin (18) PKS

(B. thailandensis MSMB43)

FR901464 (19)PKS

(Burkholderia sp. FERM BP-3421)

spliceostatin L/M (20/21) PKS

(Xanthomonas cannabis)
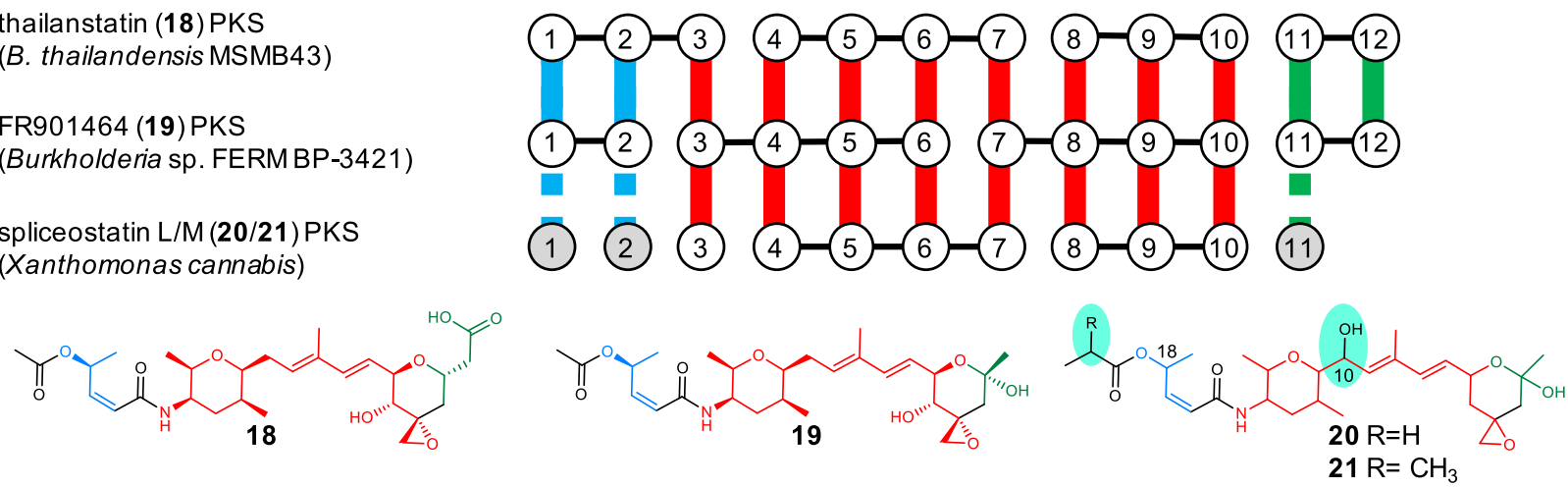

Fig. 5 Conserved motifs identified in PKSs for glutarimides, phormidolides, oocydins, and spliceostatins and their corresponding metabolites.

a Selected glutarimide-type PKSs. b Phormidolide-type and oocydin PKSs. c Spliceostatin-like PKSs. Conserved PKS module blocks and corresponding polyketide moieties are shown in the same colors. Vertical lines in PKS representations: KSs that cluster together in a phylogenetic tree; black horizontal lines: PKS protein scaffold; gray circles: PKS modules that harbor matching KS domains and domain architecture, but were identified on other, disconnected contigs in the genomic data; discontinuous vertical lines: manually assigned phylogenetic match, green highlights: structural differences compared to previously described spliceostatins. 
calc. $\left.m / z 757.4139[\mathrm{M}+\mathrm{Na}]^{+}\right)$, was close to the prediction $\left(\mathrm{C}_{41} \mathrm{H}_{64} \mathrm{O}_{11}\right)$, and hence the corresponding metabolite was selected for purification (SI Fig. 25). The purified compound was characterized by NMR as a new variant of the phormidolide scaffold that we named gynuellalide (15) (Fig. 3, SI Figs. 26-31, and SI Table 5). In comparison to the phormidolides, gynuellalide features a hemiacetal moiety at C7-C9, and lacks both the characteristic fatty acid side chain and the methyl group attached to the tetrahydrofuran ring of the phormidolides. Although the core skeleton of phormidolides and gynuellalide differ in the starter moiety around $\mathrm{C} 33 / \mathrm{C} 32$, respectively, the remaining regions as well as the KS assignments are remarkably similar, suggesting that the differences between $\mathbf{1 3}$ and $\mathbf{1 5}$ are the result of post-PKS modifications (Fig. 5b).

We were intrigued that the network node topology suggested the oocydin (ooc) PKS from Serratia plymuthica to also cluster with these PKSs, with which it shares three conserved PKS motifs spanning multiple modules (Fig. 3b). The ooc PKS generates the cytotoxin oocydin A (16) as the main product, which we recently found to be a cleaved version of the fully elongated PKS product oocydin B (17) retaining a cryptic starter moiety ${ }^{37}$. Oocydins and the cyanobacterial phormidolide-like compounds all share a core structure containing in succession: (i) an exomethylene $\beta$-branch, (ii) a tetrahydrofuran-containing moiety, and (iii) a methyl $\beta$ branch close to or at the terminus. These are interspersed with variable moieties, depending on the compound. One module block swap has resulted in the replacement by an oxygen insertion module in the ooc PKS, generating the unique internal ester moiety of 17, whereas the first module block in phormidolide-like PKSs is followed by five conserved modules that introduce a series of $\beta$-hydroxyl groups. Another large replacement of the C-terminal PKS modules accounts for the distinct macrocyclic portion of the oocydins and phormidolides.

The network and module block data suggest that these shared moieties result from a common evolutionary origin of seven modules. In the case of the cyanobacterial phor and lept PKSs, the KSs are homologous over the first 12 modules (modules 8 were assigned manually). The remaining five PKS modules in both PKSs differ in modules 13 and 15. Identification of these relationships between oocydins and the three phormidolidetype polyketides illustrates the utility of transPACT to aid not only natural product discovery, but also to identify unusual biosynthetic features. This example of mutually related patchwork-like hybrid polyketides lends further support to a general mechanism of major structural diversification by exchange of module blocks, virtually unknown for cis-AT PKSs.

Identification of spliceostatin variants from a fragmented BGC in the pathogen Xanthomonas cannabis. As a final application of transPACT, we mined the highly fragmented genome of another plant-associated bacterium for the production of a trans-AT PKSderived polyketide based on one identified conserved module block. The pathogen $X$. cannabis causes bacterial leaf spot disease in a variety of agricultural crops, characterized by necrotic lesions at the infection sites ${ }^{53-55}$. As it lacks the Hrp type III secretion system and known effectors used by many other plant-invading xanthomonads, its mechanism of pathogenicity remains unknown ${ }^{55}$. Although its draft genome is fragmented into 260 contigs, trans PACT analysis rapidly identified a trans-AT PKS section clustering with characterized spliceostatin-type PKSs (Figs. 3 and 5a). The congeners thailanstatin $A$ (18) and FR901464 (19) from the same polyketide family are promising spliceosome-inhibiting anticancer drug candidates ${ }^{56}$ previously isolated from different Burkholderia species and Pseudomonas sp. 2663, respectively 6,57 . The bioinformatic data suggested $X$. cannabis as an additional producer of spliceostatin-like polyketides. In comparison to the other spliceostatin PKSs, the $X$. cannabis PKS appeared to be incomplete, since only 8 out of the 13 spliceostatin modules were represented (Fig. 5c, SI Fig. 32, and SI Table 6). Manual analysis of contigs harboring trans-AT PKS genes enabled us to identify the core PKS BGC putatively responsible for the biosynthesis of spliceostatins (Fig. 5c). Since spliceostatins were reported to be highly potent cytotoxins ${ }^{6,57}$, we speculated that the identified PKS encodes a pathogenicity factor of $X$. cannabis.

UHPLC ESI data-dependent tandem MS in combination with molecular network analysis ${ }^{58}$ of extracts from liquid cultures of $X$. cannabis suggested the presence of more than a dozen spliceostatin analogs (SI Fig. 33). We purified two congeners (spliceostatin L (20) and spliceostatin M (21)) and characterized them by NMR (Fig. 5c, SI Figs. 34-47, and SI Tables 6 and 7), confirming their identity as additional members of this cytotoxin family. These differ from the known compounds in an a-hydroxylation at the $\mathrm{C} 10$ position, as well as in the presence of two distinct acyl ester moieties at the C18 position. 20 was tested for its ability to cause the characteristic leaf lesions using Arabidopsis thaliana as a model system. In these leaf assays, 20 caused the formation of lesions resembling the disease phenotype caused by $X$. cannabis (SI Fig. 48). Additional cytotoxicity assays with HeLa cells revealed an $\mathrm{IC}_{50}$ value of $5.9 \mathrm{nM}$ for 20 and of 1.4 nM for 21 (SI Fig. 49). These results support a role as a pathogenicity factor for spliceostatin-type compounds in $X$. cannabis.

Diversity of trans-AT PKS assembly lines in metagenomeassembled genomes. The results above were largely obtained through the analysis of genomes of cultivated bacteria. To determine whether further novelty can be found in metagenomes, we studied the functional diversity of a set of 56 trans-AT PKS BGCs that we recently identified in 20,584 metagenomeassembled genomes (MAGs) ${ }^{59}$ in the context of the diversity of known trans-AT PKS assembly lines (SI Fig. 50). Some of these assembly lines appeared to have a (virtually) identical clade composition to those responsible for the biosynthesis of known polyketides. Interestingly, these results suggest the presence of a BGC for the polyene antibiotic bacillaene, as well as an oocydinlike BGC in MAGs from the human gut, indicating that these molecules might play a role in molecular interactions in the gut microbiome. In addition, the set of MAG-derived BGCs contained several trans-AT PKS assembly lines with unprecedented functional clade compositions. Among others, these include two related complex assembly lines of $13 / 14$ modules in length that, according to GTDB-Tk ${ }^{60}$ taxonomy assignments of the associated MAGs, originate from Ruminococcus strains. Several assembly line architectures distinct from characterized PKSs were also identified in MAGs originating from other fastidious anaerobic human gut microbes, such as Blautia producta, Anaerocolumna sp., and a Lachnospiraceae bacterium to which no more detailed taxonomy could be assigned. All in all, these results show the potential of metagenome analysis to identify known trans-AT PKS systems in microbiomes of interest, as well as a way to provide new leads for discovery.

\section{Discussion}

A hallmark of polyketides produced by trans-AT PKSs is their remarkable structural diversity. In part, this diversity stems from the use of a wide array of enzymatic domains and modules that are rare or undescribed in cis-AT $\mathrm{PKSs}^{13,16}$. Here, we provide broad evidence of extensive and widespread diversification in trans-AT PKSs that results from replacement, insertion, or 
excision of conserved PKS module blocks. In a global analysis of $>1700$ trans-AT PKS systems, our transPACT analysis showed that the trans-AT PKS biosynthetic space consists of several superfamilies, within which extensive module sharing is observed. Many of these superfamilies are connected to each other by some members each sharing conserved consecutive module series with one another. Together, this strongly suggests that extensive combinatorialization and hybridization processes underlie the diversification of these polyketides. Strikingly, little evidence exists for similar mechanisms in cis-AT PKS evolution ${ }^{19}$.

Through detection of conserved module blocks and identification of cryptic relationships, transPACT aids natural product discovery in several ways. In three case studies, we demonstrate its utility as a mining tool for natural products using plantassociated bacteria as examples. In the case of gynuellalide, cryptic evolutionary relationships between phormidolide- and oocydin-type polyketides were identified. Interestingly, and despite the fact that the gynuellalide PKS is almost identical to the phormidolide PKS, the corresponding polyketide shows remarkable differences, which are likely due to extensive post-PKS modifications.

In addition to identifying cryptic evolutionary relationships between PKS families, we show that the algorithm also allows sequence-based predictions of chemical features in highly fragmented genomes. In particular for large PKS and NRPS BGCs, insufficient sequence coverage and the presence of repetitive regions in multimodular enzyme-coding genes severely compromise efforts to assemble them contiguously, which makes it difficult to predict their functions ${ }^{61}$. The automated comparative genomic analysis provided by transPACT can address this challenge. In this context, the prediction of spliceostatins from the fragmented $X$. cannabis genome yielded two potent analogs for this group of drug candidates. Their activity in leaf necrosis assays suggests an ecological function as pathogenicity factors. Other spliceosome inhibitors like herboxidiene and pladienolide were shown to exhibit herbicidal or plant growth-retardation activities $^{62,63}$. However, since the target of the new polyketides has not been experimentally verified, further studies are needed to investigate their role as spliceosome inhibitors in the context of plant pathogenesis.

Moreover, we used transPACT for the targeted expansion of the glutarimide-containing family of polyketides. The sec locus is conserved in multiple plant pathogenic bacteria and is responsible for the biosynthesis of secimide, a glutarimide-containing polyketide that resembles a truncated version of migrastatin-type polyketides. Its corresponding PKS is very similar to the migrastatin PKS over the first six modules. In comparison to the migrastatin PKS, the final four modules are missing. This observation indicates that a PKS family can be expanded through the addition or deletion of module blocks from preexisting, functional PKSs and the resulting PKSs remain functional.

The identification of conserved PKS module blocks that correspond to shared polyketide moieties enhances our understanding of natural product evolution, and suggests implications for drug discovery and PKS engineering. Why is module block swapping prevalent in trans-AT PKSs? Exchange of larger structural moieties provides a strong force of polyketide innovation compared to module duplication as known for cis-AT PKSs, thus permitting polyketide evolution "in quantum leaps". Such polyketide moieties might be maintained and combinatorially used for structural reasons, such as permitting closure of (macrocyclic) rings, mediating recognition of the metabolite by exporters, or conferring pharmacological activity. Support for pharmacophores as selection forces for module blocks is provided by actin-binding macrolides, which use an exocyclic tail moiety for target binding ${ }^{64}$ that recurs as a combinatorial-like unit in several otherwise unrelated congeners ${ }^{29}$. In the diverse family of pederin-type polyketides, a large shared moiety confers cytotoxic activity, while swapped parts further modulate the bioactivity profile, including potent dermatotoxicity only in congeners with lipophilic termini ${ }^{65}$. It would be worthwhile to test whether the conserved tetrahydrofuran portion in the cytotoxic oocydins and phormidolides are likewise determinants of bioactivity. Extrapolated to natural product mining, the use of transPACT to target uncharacterized but widely conserved module blocks could be an interesting rationale to discover evolutionarily successful bioactive principles in nature. We have made transPACT available as an open-source software, which scientists can apply on their own custom (meta)genomic datasets ${ }^{66}$.

In addition to keeping a pharmacophore intact, preserved module blocks could be important at the biochemical level as the successful recombination of module blocks might frequently result in functional chimeric PKSs. transPACT revealed dozens of such putative successful recombination events. The extent of module block recombination is remarkable, as putatively hybridized PKS assembly lines are observed across phylum borders in numerous instances. However, we cannot exclude that recombination occurred between ancestral clusters present in the same source taxon, followed by transfer of whole clusters to other phyla. Previous studies by one of our labs has located recombination sites in trans-AT systems behind KS domains ${ }^{29,31}$, supporting the redefinition of a biosynthetic module as also proposed by others for cis-AT PKSs ${ }^{67,68}$. Classically, the KS and ACP domains have been defined as module boundaries. From an evolutionary and biochemical perspective, however, a module would more logically start downstream of an (in trans-AT PKSs often substrate-specific) KS domain and end with the next KS, which phylogenetically and biochemically matches the modification introduced by this module ${ }^{68}$. Recombination between different trans-AT PKSs behind specific KSs would be expected to more likely result in functional PKSs than for traditional module boundaries. Identifying the exact recombination loci as well as focusing on evolutionarily successful module blocks will provide important clues for rationally engineering functional hybrid trans-AT PKSs by module block fusion. Moreover, rational excisions and insertions could be designed once the exact locations of functional recombination events have been defined.

In summary, our analysis groups trans-AT PKSs into three major categories. The first category consists of PKSs that are conserved in a variety of different producers and putatively responsible for the biosynthesis of identical or closely related polyketides. While this result shows the potential of transPACT to conduct in silico dereplication studies even for fragmented (meta)genomes, it also offers the possibility to identify cultured sources for polyketides initially isolated from uncultured bacteria (i.e., luminaolide $)^{26,29}$. This strategy could be relevant for biotechnological production, since many bioactive trans-AT PKS products have been isolated from uncultured sources ${ }^{8,13}$. The second PKS category comprises enzymes that harbor a conserved module block corresponding to a shared polyketide substructure. Targeting these polyketides might lead to the identification of polyketides with pharmacophores within otherwise highly dissimilar scaffolds, such as in the case of the glutarimide polyketides studied here. Thirdly, the existence of common motifs present only in orphan PKSs (unlabeled node groups in Fig. 2) point to a large potential to identify biosynthetic diversity in future genome mining studies.

\section{Methods}

Trans-AT PKS assembly line identification, annotation, and phylogenetic

analysis. The set of characterized trans-AT PKS assembly lines ${ }^{16,17}$ was manually gathered from $\mathrm{MIBiG}^{69}$ and literature; the set of predicted trans-AT PKS assembly 
lines was gathered from antiSMASH ${ }^{38}$ results against the entirety of GenBank (version of December 2015). KS domains in all assembly lines were predicted using the SMART ${ }^{70}$-derived PKS_KS.hmm domain, using HMMer v2 instead of v3 to avoid undue gaps inside the hits. Domains from cis-AT modules were filtered out by checking for hits with the PKS_AT.hmm model. Domains were aligned using Muscle in MEGA v6 and a phylogenetic tree was constructed with RAxML, under a GTRGAMMA substitution model. Functional clades in the reference tree were assigned manually, and phylogenetic placement of predicted KS domains was performed using pplacer v1.1.6 $6^{33}$.

\section{Prediction of the order of KS domains across PKS assembly lines. For KS} domains that are exclusively encoded on the positive or negative strand, the KS domains were ordered according to their genomic location (1228 BGCs are encoded exclusively on the same strand orientation). For KS domains that are encoded on both positive (+) and negative strands (-), we separated them into two situations: partial mixture and complete mixture. Partial mixture describes the situation where all domains encoded on positive strands have a genomic location larger (or smaller) than the largest (or smallest) genomic location of those on the negative strands $(---+++$ or +++---$)$. Complete mixture is the situation where at least one domain on the positive strand is located in between the ones on the negative strand or vice versa $(--+-++)$. For partial mixture, if a TE domain, which is considered to terminate biosynthesis, is identified and the TE domain is located next to the terminal KS domain on one strand, the biosynthesis is considered to start from the KS domains on the other strand (times of correction: 91 TEs on negative strand, 96 TEs on positive strand). If no TE domain is identified, the KS domains on the two strands are indexed separately. For complete mixture, which only happened three times, we did not index the KS domains. TE domains are identified with Pfam TC trusted threshold cutoffs in HMMER v2.

Organisms and culture conditions. Seed cultures of Xanthomonas campestris pv. cannabis (X. cannabis) were prepared by inoculating $10 \mathrm{~mL}$ peptone-sucrose (PS) medium in baffled Erlenmeyer flasks from cryo-stocks of X. cannabis. After cultivation at $30^{\circ} \mathrm{C}$ overnight at 200 rounds per minute (r.p.m.), $1 \mathrm{~L}$ baffled Erlenmeyer flasks $(17 \times)$ containing $200 \mathrm{~mL}$ PS medium were inoculated with $400 \mu \mathrm{L}$ of seed culture. Bacteria were incubated at $30^{\circ} \mathrm{C}$ for $40 \mathrm{~h}$ at 200 r.p.m. Pseudomonas syringae B728a was cultured in SR-minimal medium supplemented with $0.1 \%$ fructose and $100 \mu \mathrm{M}$ arbutin in baffled Erlenmeyer flasks at $20^{\circ} \mathrm{C}$ for 4 days. Gynuella sunshinyii was cultured in marine broth 2216 in $1 \mathrm{~L}$ ultrahigh yield flasks for 3 days at $30^{\circ} \mathrm{C}$ at 120 r.p.m.

Construction of a secimide production deficient mutant. An unmarked deletion mutant of Psyr_4314 was constructed as described previously ${ }^{71}$. Briefly, the $5^{\prime}$ and $3^{\prime}$ regions flanking Psyr_4314 were amplified in a first round of PCR reactions, in addition to a kanamycin resistance cassette flanked with FRT sites from pKD13.8 The primers 4314-F1 and 4314-R1 were used to amplify the region upstream of Psyr_4314, while 4314-F2 and 4314-R2 were used to amplify the region downstream of Psyr 4314, and FRT-KM-F and FRT-KM-R were used to amplify the FRTflanked kanamycin resistance cassette (Supplementary Table 9). In a subsequent PCR, all three fragments were combined and amplified for 15 cycles without added primers, followed by addition of 4314-F1 and 4314-R2 for 20 more PCR cycles to amplify the combined fragment. The resulting PCR product was cloned into the suicide vector $\mathrm{pTOK} 2 \mathrm{~T} 9$. The obtained construct was subsequently transferred into P. syringae by tri-parental mating ${ }^{10}$ and transformants selected on KB plates containing rifampin, kanamycin, and tetracycline. Double crossover mutants as indicated by kanamycin resistance, but tetracycline sensitivity were subsequently identified and the kan cassette excised. Excising was conducted by introducing plasmid pFLP211 in which the omega fragment conferring spectinomycin resistance had been added. Replica plating was used to cure the $\Delta 4314$ strain of pFLP2-omega. Successful markerless deletions were confirmed by PCR.

Detection of secimide. A total of $200 \mathrm{~mL}$ cultures of $P$. syringae B728a WT and a $s e c$ knockout strain were harvested by centrifugation. The supernatants of both cultures were extracted with EtOAc, and the organic layers concentrated in vacuo. The pellet was extracted with acetone, and also concentrated in vacuo. All extracts were dissolved in $\mathrm{MeOH}$, and subjected to HR-LCMS analysis $(5 \% \mathrm{MeCN}$ for $5 \mathrm{~min}, 5-100 \% \mathrm{MeCN}$ gradient for $30 \mathrm{~min}$, washed with $100 \% \mathrm{MeCN}$, column: Phenomenex Kinetex $2.6 \mu \mathrm{m}$ XB-C18 $100 \AA, 4.6 \times 150 \mathrm{~mm}$, UV detection at $\lambda=$ $220 \mathrm{~nm}$, column oven: $27^{\circ} \mathrm{C}$ ). The most significant difference between WT and knockout in the LCMS chromatogram was attributed to one compound with the $\mathrm{m} / \mathrm{z}$ of 326.16 eluting after $16-18 \mathrm{~min}$ that only appeared in the WT supernatant.

Isolation and structure elucidation of secimide. A $1 \mathrm{~L}$ culture of $P$. syringae B728a was centrifuged, and the supernatant was extracted with EtOAc. The organic layer was concentrated in vacuo, and separated by reversed-phase HPLC (Phenomenex Luna $5 \mu \mathrm{m} \mathrm{C} 18,10 \times 250 \mathrm{~mm}$, UV detection at $l=220 \mathrm{~nm}$, room temperature (r.t.)) eluted with $5 \% \mathrm{MeCN}$ for $5 \mathrm{~min}$, then a gradient from $5 \% \mathrm{MeCN}$ to $100 \% \mathrm{MeCN}$ for $30 \mathrm{~min}$, and $100 \% \mathrm{MeCN}$ for $25 \mathrm{~min}$. The fraction containing secimide was further separated by reversed-phase HPLC (Phenomenex Luna $5 \mu \mathrm{m}$
Phenylhexyl, $10 \times 250 \mathrm{~mm}$, UV detection at $\lambda=220 \mathrm{~nm}$, r.t.) eluted with $20 \%$ $\mathrm{MeCN}$ to afford $0.4 \mathrm{mg}$ of secimide.

Secimide has a molecular formula of $\mathrm{C}_{16} \mathrm{H}_{23} \mathrm{NO}_{6}$, which was suggested by $\mathrm{HR}-$ LC-ESIMS ( $m / z$ 326.1597, $[\mathrm{M}+\mathrm{H}]^{+}, \Delta-0.1 \mathrm{~m}$.m.u.). Analysis of the ${ }^{1} \mathrm{H}$ NMR spectrum in conjunction with the HSQC data revealed the presence of one protonated $\mathrm{sp}^{2}$ carbon, one methoxymethyl, one oxygenated methine, one doublet methyl, one vinyl methyl, two methines and four methylenes. Unit I and unit II were determined by COSY analysis. The HMBC correlations from $\mathrm{H} 7, \mathrm{H} 10$, and H11 to C8 suggested that unit I was connected to the methyl ester moiety. The $\mathrm{HMBC}$ correlations from $\mathrm{H} 6$ to $\mathrm{C} 9$, and from $\mathrm{H} 9$ to $\mathrm{C} 5$ and $\mathrm{C} 6$ suggested that the vinyl methyl group was connected to unit I. Unit II and this vinyl methyl group were connected via a ketone carbon (C4) based on the HMBC correlations from $\mathrm{H} 3$, $\mathrm{H} 9$, and $\mathrm{H} 6$ to $\mathrm{C} 4$. The piperidine-2,6-dione moiety was determined based on the $\mathrm{HMBC}$ correlations from $\mathrm{H}^{\prime}$ to $\mathrm{C}^{\prime}$ and $\mathrm{C}^{\prime}$, from $\mathrm{H}^{\prime}$ to $\mathrm{C}^{\prime}$ and $\mathrm{C}^{\prime}$, as well as the molecular formula.

The $5 E$ geometry of secimide was determined by NOESY correlations between $\mathrm{H} 7$ and H9. The absolute stereochemistry of $\mathrm{C} 2$ was determined as $R$ by applying the modified Mosher's method.

Preparation of secimide MTPA esters. A total of $1 \mathrm{mg}$ of secimide was added to $S$-(+)- or $R$-(-)-MTPACl $(5 \mu \mathrm{L})$ in $100 \mu \mathrm{L}$ of $\mathrm{CH}_{2} \mathrm{Cl}_{2}$ containing $1 \mathrm{mg}$ of DMAP for $2 \mathrm{~h}$ at $40{ }^{\circ} \mathrm{C}$. The mixture was partitioned between $0.1 \mathrm{M} \mathrm{NaHCO}_{3}$ and $\mathrm{CHCl}_{3}$. The organic layer was washed with $0.1 \mathrm{M} \mathrm{HCl}$ and $\mathrm{H}_{2} \mathrm{O}$, and then concentrated in vacuo. The extract was separated by reversed-phase HPLC (Phenomenex Luna $5 \mu \mathrm{m} \mathrm{C18}, 10 \times 250 \mathrm{~mm}$, UV detection at $\lambda=220 \mathrm{~nm}$, r.t.) eluted with $5 \% \mathrm{MeCN}$ for $5 \mathrm{~min}$, then a gradient from $5 \% \mathrm{MeCN}$ to $100 \% \mathrm{MeCN}$ for $30 \mathrm{~min}$, and $100 \%$ $\mathrm{MeCN}$ for $25 \mathrm{~min}$ to afford $R-(+)$ - or $S$-(-)-MTPA esters, respectively.

Isolation and structure elucidation of gynuellalide. A $10 \mathrm{~L}$ culture of $\mathrm{G}$. sunshinyii was centrifuged and the supernatant extracted with EtOAc. The extract was concentrated in vacuo, and the crude extract was separated by reversed-phase HPLC (Phenomenex Luna $5 \mu \mathrm{m} \mathrm{C18}, 20 \times 250 \mathrm{~mm}$, UV detection at $l=200 \mathrm{~nm}$, r.t.), using the following gradient: $5 \% \mathrm{MeCN}$ for $5 \mathrm{~min}$, then a gradient from $5 \% \mathrm{MeCN}$ to $100 \% \mathrm{MeCN}$ for $30 \mathrm{~min}$, and $100 \% \mathrm{MeCN}$ for $25 \mathrm{~min}$. The fractions containing gynuellalide were further separated by reversed-phase HPLC (Phenomenex Synergi Hydro-RP $5 \mu \mathrm{m} \mathrm{C18}, 10 \times 250 \mathrm{~mm}$, UV detection at $\lambda=200 \mathrm{~nm}$, r.t.), using an isocratic solvent composition of $30 \% \mathrm{MeCN}$. Purification was achieved by reversedphase HPLC (Phenomenex Luna $5 \mu \mathrm{m}$ Phenylhexyl, $10 \times 250 \mathrm{~mm}$, UV detection at $\lambda=200 \mathrm{~nm}$, r.t.) using an isocratic elution profile with $35 \% \mathrm{MeCN}+0.1 \% \mathrm{FA}$ to afford $0.3 \mathrm{mg}$ of gynuellalide.

Gynuellalide had a molecular formula of $\mathrm{C}_{16} \mathrm{H}_{23} \mathrm{NO}_{6}$, which was suggested by HR-LC-ESIMS $\left(\mathrm{m} / z 757.4147[\mathrm{M}+\mathrm{Na}]^{+}, \Delta+1.3\right.$ m.m.u.). Analysis of the ${ }^{1} \mathrm{H}$ NMR spectrum in conjunction with the HSQC data revealed the presence of one exomethylene, two singlet aliphatic methyls, one doublet aliphatic methyl, three vinylic methyls, eight oxygenated methines, one dioxygenated methine, and three protonated sp2 carbons. COSY spectrum deduced the partial structures I-VII. Unit I and unit II was connected by HMBC correlations from H39 to C25 and from $\mathrm{OH} 25$ to C26. Unit II was connected to unit III via a dimethyl quaternary carbon, which was determined by $\mathrm{HMBC}$ correlations from $\mathrm{OH} 21$ to $\mathrm{C} 22$, from $\mathrm{H} 23$ to $\mathrm{C} 21$, from $\mathrm{OH} 23$ to $\mathrm{C} 22$, from $\mathrm{H} 37$ to $\mathrm{C} 21, \mathrm{C} 22, \mathrm{C} 23$ and $\mathrm{C} 38$, and from $\mathrm{H} 38$ to C21, C22, C23, and C37. Unit III and unit IV was connected by HMBC correlations from $\mathrm{H} 18$ to $\mathrm{C} 20$, from $\mathrm{H} 20$ to $\mathrm{C} 18, \mathrm{C} 19$, and $\mathrm{C} 20$, and from $\mathrm{H} 36$ to C20. The ether ring in unit IV was determined by a weak HMBC correlation from $\mathrm{H} 13$ to $\mathrm{C} 16$. Unit IV and unit $\mathbf{V}$ were connected by HMBC correlations from $\mathrm{H} 10$ to $\mathrm{C} 12$, from $\mathrm{H} 12$ to $\mathrm{C} 11$ and $\mathrm{C} 35$, and from $\mathrm{H} 35$ to $\mathrm{C} 12$. A hemiacetal moiety formed by unit $\mathbf{V}$ and unit VI was supported by HMBC correlations from $\mathrm{H} 8$ to $\mathrm{C} 34$, from $\mathrm{H} 9$ to $\mathrm{C} 34$, from $\mathrm{H} 34$ to $\mathrm{C} 7, \mathrm{C} 8$, $\mathrm{C}$, and $\mathrm{C} 10$, and from $\mathrm{OH} 34$ to $\mathrm{C} 9$. Unit $\mathbf{V}$ was connected to unit VII by HMBC correlations from $\mathrm{H} 2$ to $\mathrm{C} 4$, from $\mathrm{H} 4$ to $\mathrm{C} 2, \mathrm{C} 3$, and $\mathrm{C} 33$, from $\mathrm{H} 5$ to $\mathrm{C} 3$, and from $\mathrm{H} 33$ to $\mathrm{C} 4$. The macrolide was formed between unit IV and unit VII by HMBC correlations from H2 and H15 to $\mathrm{C}$. Finally, the five-membered lactone moiety with the exomethylene group was attached to the terminus of unit $\mathbf{I}$, as determined by the molecular formula and HMBC correlations from $\mathrm{H} 30$ to $\mathrm{C} 31, \mathrm{C} 32$, and $\mathrm{C} 40$, and from $\mathrm{H} 40$ to $\mathrm{C} 30$ and $\mathrm{C} 32$.

The $2 E, 10 E, 18 E$ geometry was determined by ROESY correlations between $\mathrm{H} 2$ and $\mathrm{H} 4$, between $\mathrm{H} 9$ and $\mathrm{H} 35$, between $\mathrm{H} 10$ and $\mathrm{H} 12$, and between $\mathrm{H} 17$ and $\mathrm{H} 36$, The relative stereochemistry of $\mathrm{C} 7$ and $\mathrm{C} 9$ was determined as $7 R^{\prime}, 9 R^{\prime}$ by ROESY correlations between $\mathrm{H} 6$ and H7a, between $\mathrm{H} 7 \mathrm{a}$ and H10, and between $\mathrm{H} 6$ and $\mathrm{H} 10$. The relative stereochemistry of the ether ring was determined as $13 S^{\prime}, 15 S^{\prime}, 16$ $R^{\prime}$ by ROESY correlations between $\mathrm{H} 12$ and H15, between $\mathrm{H} 12$ and H17, between $\mathrm{H} 13$ and H16, and between $\mathrm{H} 15$ and H17.

Isolation of spliceostatins. Xanthomonas cannabis cultures were incubated at $30^{\circ} \mathrm{C}$ for $40 \mathrm{~h}$ at 200 r.p.m. After centrifugation, the highly viscous supernatant was extracted once with $80 \%(\mathrm{v} / \mathrm{v})$ EtOAc/water, then with $40 \%(\mathrm{v} / \mathrm{v})$ EtOAc/water. The organic layers were combined and the solvent removed under reduced pressure to yield $250 \mathrm{mg}$ of crude extract. The residue was dissolved in $2 \mathrm{~mL}$ of acetonitrile and stored at $-20^{\circ} \mathrm{C}$. The precipitate was removed once a day for 5 days. The clear solution was purified on an Agilent 1260 Infinity HPLC using a Kinetex C18 $100 \AA$ 
column (mobile phase A water, mobile phase B acetonitrile; flow rate $2.0 \mathrm{~mL} / \mathrm{min}$; gradient, $5-35 \%$ B for $8 \mathrm{~min}, 35-95 \%$ B for $32 \mathrm{~min}, 95 \%$ B for $8 \mathrm{~min}, 95-5 \%$ B for $3 \mathrm{~min}, 5 \% \mathrm{~B}$ for $1 \mathrm{~min}$ ). The collected fractions were analyzed on a Thermo Orbitrap UPLC-HESI-MS. A total of $0.2 \mathrm{mg}$ of $\mathbf{2 0}$ and $0.2 \mathrm{mg}$ of $\mathbf{2 1}$ were obtained and the structures characterized by $1 \mathrm{D}$ and $2 \mathrm{D}$ NMR experiments.

\section{Structure elucidation of spliceostatin M (21). The molecular formula of} $\mathrm{C}_{29} \mathrm{H}_{45} \mathrm{NO}_{8}$ was predicted based on HR-ESIMS spectroscopy. The HMBC correlations from $\mathrm{H} 2$ and $\mathrm{H} 17$ to the chemical shift of 97.2 p.p.m. characteristic for an acetal, together with the HMBC correlations from the alcohol at 3.71 to $\mathrm{C} 1, \mathrm{C} 2$, and $\mathrm{C} 17$, were used to determine the hydroxylation of $\mathrm{C} 1$. Based on HMBC correlations from $\mathrm{H} 2$ to $\mathrm{C} 3$ and $\mathrm{H} 18$ to $\mathrm{C} 2, \mathrm{C} 3$, and $\mathrm{C} 4$ the epoxide was connected. The coupling constant of $\mathrm{H} 6$ and $\mathrm{H} 7(3 \mathrm{JH}-6, \mathrm{H}-7=15.8 \mathrm{~Hz})$ suggests trans-configuration. The NOESY spectra shows cross peaks between $\mathrm{H} 9$ and $\mathrm{H} 7$, as well as $\mathrm{H} 10$ and $\mathrm{H} 19$ suggesting trans-configuration for the double bond between $\mathrm{C} 8$ and C9. The COSY correlation of the alcohol at 2.95 to $\mathrm{H} 10$ and the HMBC correlation of it to $\mathrm{C} 9, \mathrm{C} 10$, and $\mathrm{C} 11$ suggest hydroxylation of $\mathrm{C} 10$. $\mathrm{C} 12$ and $\mathrm{C} 13$ were connected by $\mathrm{HMBC}$ correlations of their respective protons. $\mathrm{HMBC}$ correlations of $\mathrm{NH}$ toward $\mathrm{Cl}^{\prime}$ and $\mathrm{C} 14$ connect the two parts of the molecule through a peptide bond. The coupling constant of $\mathrm{H}^{\prime}$ and $\mathrm{H}^{\prime}{ }^{\prime}\left(3 \mathrm{JH}-2^{\prime}, \mathrm{H}-3^{\prime}=11.7 \mathrm{~Hz}\right)$ suggests cisconfiguration. $\mathrm{HMBC}$ correlations of $\mathrm{H}^{\prime}$ to $\mathrm{C}^{\prime \prime}$ connect the isobutyrate group to the molecule. The relative stereochemistry of the Western pyran ring was determined by analysis of the NOESY spectrum. The axial protons H11 and H15 show cross peaks, as well as $\mathrm{NH}$ with $\mathrm{C} 16$ and $\mathrm{C} 20$. The relative stereochemistry of the Eastern pyran ring could not be resolved due to a lack of correlation signals between $\mathrm{H} 2$ and $\mathrm{H} 4$.

Structure elucidation of spliceostatin L (20). The molecular formula of $\mathrm{C}_{28} \mathrm{H}_{43} \mathrm{NO}_{8}$ was predicted based on HR-ESIMS spectroscopy. The HMBC correlations from $\mathrm{H} 2$ and $\mathrm{H} 17$ to the for an acetal characteristic chemical shift of 97.2 ppm together with the HMBC correlations from the alcohol at 3.72 to $\mathrm{C} 1, \mathrm{C} 2$, and $\mathrm{C} 17$ were used to determine the hydroxylation of $\mathrm{C} 1$. Based on HMBC correlations from $\mathrm{H} 2$ to $\mathrm{C} 3$, and $\mathrm{H} 18$ to $\mathrm{C} 2, \mathrm{C} 3$, and $\mathrm{C} 4$, the epoxide was connected. The coupling constant of $\mathrm{H} 6$ and $\mathrm{H} 7(3 \mathrm{JH}-6, \mathrm{H}-7=15.8 \mathrm{~Hz})$ suggests trans-configuration. The NOESY spectra shows cross peaks between $\mathrm{H} 9$ and $\mathrm{H} 7$ as well as $\mathrm{H} 10$ and $\mathrm{H} 19$ suggesting trans-configuration for the double bond between $\mathrm{C} 8$ and $\mathrm{C}$. The COSY correlation of the alcohol at 2.95 to $\mathrm{H} 10$ and the HMBC correlation of it to $\mathrm{C} 9, \mathrm{C} 10$, and $\mathrm{C} 11$ suggest hydroxylation of $\mathrm{C} 10$. $\mathrm{C} 12$ and $\mathrm{C} 13$ were connected by HMBC correlations of their respective protons. HMBC correlations of NH toward $\mathrm{Cl}^{\prime}$ and $\mathrm{C} 14$ connect the two parts of the molecule through a peptide bond. The coupling constant of $\mathrm{H}^{\prime}{ }^{\prime}$ and $\mathrm{H} 3^{\prime}\left(3 \mathrm{JH}-2^{\prime}, \mathrm{H}-3^{\prime}=11.7 \mathrm{~Hz}\right)$ suggests cis-configuration. $\mathrm{HMBC}$ correlations of $\mathrm{H}^{\prime}$ to $\mathrm{Cl}^{\prime \prime}$ connect the propionate group to the molecule. The relative stereochemistry of the Western pyran ring was determined by analysis of the NOESY spectrum. The axial protons H11 and H15 show cross peaks, as well as NH with C16 and C20. The relative stereochemistry of the Eastern pyran ring could not be resolved due to a lack of correlation signals between $\mathrm{H} 2$ and $\mathrm{H} 4$.

Instrumentation and software. NMR spectra were recorded on a Bruker Avance III spectrometer equipped with a cold probe at 500 or $600 \mathrm{MHz}$ for ${ }^{1} \mathrm{H} \mathrm{NMR}$, and 125 or $150 \mathrm{MHz}$ for ${ }^{13} \mathrm{C}$ NMR. Chemical shifts were referenced to the solvent peak at $\delta_{\mathrm{H}} 7.27$ and $\delta_{\mathrm{C}} 77.23$ for chloroform- $d, \delta_{\mathrm{H}} 2.50$ and $\delta_{\mathrm{C}} 39.51$ for DMSO- $d 6$, and $\delta_{\mathrm{H}} 1.94$ and $\delta_{\mathrm{C}} 1.39$ for acetonitrile- $d 3$. UHPLC-HRESI-MS was performed on a Thermo Scientific Q Exactive mass spectrometer. Software versions used for data analysis are Xcalibur 4.1 (Thermo) and Chromeleon Xpress version 7.2 (Thermo) for MS, Open Lab CDS version 2.2 (Agilent) for HPLC, and TopSpin version 3.5.7 (Bruker) for NMR.

Cultivation of A. thaliana. Arabidopsis thaliana Col-0 seeds were prepared as described by Innerebner et al. ${ }^{72}$. Plants were cultivated in full-gas 24 -well plates on Murashige Skoog-medium 9 (MS-medium) at $22{ }^{\circ} \mathrm{C}$ in a climate chamber. After 1 week under long-day conditions ( $16 \mathrm{~h}$ photoperiod) the photoperiod was reduced to short-day conditions ( $9 \mathrm{~h}$ photoperiod).

Inoculation of $\boldsymbol{A}$. thaliana with $\mathbf{X}$. cannabis and spliceostatins. Xanthomonas campestris pv. cannabis was grown in PS medium overnight. Cells were harvested and the pellet was washed once with water, resuspended in water, and diluted to an optical density of $\mathrm{OD}_{600}$ of 0.5 . 20 was dissolved in $10 \%$ acetonitrile in water to obtain a concentration of $1.8 \mathrm{mM}$. Solutions with the following concentrations were prepared $180,18,1.8$, and $0.18 \mu \mathrm{M}$ by dilution with water. In 24 -well plates, $1 \mu \mathrm{L}$ of each solution was spotted onto one leaf of three plants, respectively, water was chosen as a negative control, X. cannabis suspension as a positive control. The experiment was performed on three independent 24-well plates. After inoculation with bacteria and treatment with $\mathbf{2 0}$, the plants were cultivated under short-day conditions ( $9 \mathrm{~h}$ photoperiod). Pictures were taken directly after inoculation ( 0 d.p.i.) and 10 days post inoculation (10 d.p.i.).
HeLa cell cytotoxicity assay. HeLa cells were cultivated at $37^{\circ} \mathrm{C}, 5 \%(\mathrm{v} / \mathrm{v}) \mathrm{CO}_{2}$ for 3-4 days. HeLa cells were washed twice with PBS buffer (Sigma D8537), $2 \mathrm{~mL}$ of 0.05\% trypsin-EDTA solution (Thermo 25300-054) were added, and the plate was incubated for $5 \mathrm{~min}$ at $37^{\circ} \mathrm{C}$. The cells were resuspended in $10 \mathrm{~mL}$ medium (DMEM-GlutaMAX) supplemented with 10\% FCS (Eurobio CVFSVF00-01), 1\% nonessential amino acids (Thermo 11140-035), and $50 \mu \mathrm{g} / \mathrm{mL}$ gentamicin and centrifuged for $5 \mathrm{~min}, 110$ r.c.f. at r.t. The supernatant was discarded and the pellet resuspended in $10 \mathrm{~mL}$ medium. Cells were counted by analyzing $10 \mu \mathrm{L}$ of the suspension under a ZEISS Axiovert 25 microscope using a Neubauer hemocytometer. A 5000 cells $/ \mathrm{mL}$ suspension was prepared and $200 \mu \mathrm{L}$ were transferred into each well of a 96-well plate. After 3 days of cultivation, $2 \mu \mathrm{L}$ sample were added to the lanes B2-11. DMSO was used as a negative control, doxorubicin $(c=1 \mathrm{mg} / \mathrm{mL}$ in DMSO) as a positive control. Both were measured in triplicates. Isolated spliceostatin L (20) and spliceostatin M (21) were used at a concentration of $c=3 \mu \mathrm{M}$ in DMSO in quadruplicates. A total of $50 \mu \mathrm{L}$ medium were added to the wells in row $B$, mixed by pipetting and transferred to the next row. This procedure was repeated until row $\mathrm{G}$, thereby diluting the concentration of the samples five times. After 3 days of cultivation, $50 \mu \mathrm{L}$ of 3-(4,5-dimethylthiazol-2-yl)-2,5-diphenyltetrazolium bromide $\left(1 \mathrm{mg} / \mathrm{mL}\right.$ in sterile $\left.\mathrm{H}_{2} \mathrm{O}\right)$ were added and the cells incubated for $3 \mathrm{~h}$ at $37^{\circ} \mathrm{C}$. The supernatant was discarded and $150 \mu \mathrm{L}$ of DMSO were added to the wells. The absorbance at $570 \mathrm{~nm}$ was measured on a spectraMAXplus spectrometer (Molecular Devices LLC). A 96-well plate with DMSO served as a blank, all measurements were performed in technical triplicates. Relative and absolute $\mathrm{IC}_{50}$ values were calculated by fitting a four-parameter logistic model to the mean of the biological replicates in GraphPad Prism 7. For determination of the absolute $\mathrm{IC}_{50}$, data were normalized by averaging the absorbance values measured at highest doxorubicin concentrations and setting it as the bottom of the curve (bottom constrained to 0.0 ), and the three negative control values containing the least DMSO and setting it as the top of the curve (top constrained to 1.0).

Reporting summary. Further information on research design is available in the Nature Research Reporting Summary linked to this article.

\section{Data availability}

GenBank: the spliceostatin gene cluster from X. cannabis was deposited in GenBank under accession number BK010647. MIBiG: the spliceostatin, gynuellalide, and secimide BGCs were deposited in MIBiG under accession numbers BGC0002059, BGC0001835, and BGC0002060. All other raw data are available from the corresponding authors upon request. Source data are provided with this paper.

\section{Code availability}

The transPACT source code is available freely under an open-source AGPL-3.0 license from https://github.com/chevrm/transPACT (https://doi.org/10.5281/zenodo.4148258).

Received: 13 June 2020; Accepted: 6 January 2021;

Published online: 03 March 2021

\section{References}

1. Pappa, K. A. The clinical development of mupirocin. J. Am. Acad. Dermatol. 22, 873-879 (1990).

2. Mast, Y. et al. Characterization of the 'pristinamycin supercluster' of Streptomyces pristinaespiralis. Microb. Biotechnol. 4, 192-206 (2011).

3. Pettit, G. R. From a marine bryozoan to the clinic: the bryostatins. Abstr. Pap. Am. Chem. Soc. 223, 128 (2002)

4. Schäberle, T. F. et al. Corallopyronin A - a promising antibiotic for treatment of filariasis. Int. J. Med. Microbiol. 304, 72-78 (2014).

5. Nakae, K. et al. Migrastatin, a new inhibitor of tumor cell migration from Streptomyces sp. MK929-43F1. Clin. Cancer Res. 6, 299 (2000).

6. Eustaquio, A. S., Janso, J. E., Ratnayake, A. S., O’Donnell, C. J. \& Koehn, F. E Spliceostatin hemiketal biosynthesis in Burkholderia spp. is catalyzed by an iron/a-ketoglutarate-dependent dioxygenase. Proc. Natl Acad. Sci. USA 111, 3376-3385 (2014).

7. Kwan, J. C. et al. Genome streamlining and chemical defense in a coral reef symbiosis. Proc. Natl Acad. Sci. USA 109, 20655-20660 (2012).

8. Piel, J. Metabolites from symbiotic bacteria. Nat. Prod. Rep. 26, 338-362 (2009).

9. Rust, M. et al. A multiproducer microbiome generates chemical diversity in the marine sponge Mycale hentscheli. Proc. Natl. Acad. Sci. U.S.A. 117, 9508-9518 (2020).

10. Biggins, J. B., Ternei, M. A. \& Brady, S. F. Malleilactone, a polyketide synthase-derived virulence factor encoded by the cryptic secondary metabolome of Burkholderia pseudomallei group pathogens. J. Am. Chem. Soc. 134, 13192-13195 (2012). 
11. Franke, J., Ishida, K. \& Hertweck, C. Genomics-driven discovery of burkholderic acid, a noncanonical, cryptic polyketide from human pathogenic Burkholderia species. Angew. Chem. Int. Ed. Engl. 51, 11611-11615 (2012).

12. Möbius, N. et al. Biosynthesis of the respiratory toxin bongkrekic acid in the pathogenic bacterium Burkholderia gladioli. Chem. Biol. 19, 1164-1174 (2012).

13. Helfrich, E. J. \& Piel, J. Biosynthesis of polyketides by trans-AT polyketide synthases. Nat. Prod. Rep. 33, 231-316 (2016).

14. O’Brien, R. V., Davis, R. W., Khosla, C. \& Hillenmeyer, M. E. Computational identification and analysis of orphan assembly-line polyketide synthases. $J$. Antibiot. 67, 89-97 (2014).

15. Blin, K., Medema, M. H., Kottmann, R., Lee, S. Y. \& Weber, T. The antiSMASH database, a comprehensive database of microbial secondary metabolite biosynthetic gene clusters. Nucleic Acids Res. 45, 555-559 (2017).

16. Piel, J. Biosynthesis of polyketides by trans-AT polyketide synthases. Nat. Prod. Rep. 27, 996-1047 (2010).

17. Helfrich, E. J. N. et al. Automated structure prediction of trans-acyltransferase polyketide synthase products. Nat. Chem. Biol. 15, 813-821 (2019).

18. Jenke-Kodama, H., Börner, T. \& Dittmann, E. Natural biocombinatorics in the polyketide synthase genes of the actinobacterium Streptomyces avermitilis. PLoS Comput. Biol. 2, 1210-1218 (2006).

19. Wlodek, A. et al. Diversity oriented biosynthesis via accelerated evolution of modular gene clusters. Nat. Commun. 8, 1206 (2017).

20. Medema, M. H., Cimermancic, P., Sali, A., Takano, E. \& Fischbach, M. A. A systematic computational analysis of biosynthetic gene cluster evolution: lessons for engineering biosynthesis. PLoS Comput. Biol. 10, e1004016 (2014).

21. Jenke-Kodama, H., Sandmann, A., Müller, R. \& Dittmann, E. Evolutionary implications of bacterial polyketide synthases. Mol. Biol. Evol. 22, 2027-2039 (2005).

22. Nguyen, T. et al. Exploiting the mosaic structure of trans-acyltransferase polyketide synthases for natural product discovery and pathway dissection. Nat. Biotechnol. 26, 225-233 (2008).

23. Chevrette, M. G. \& Currie, C. R. Emerging evolutionary paradigms in antibiotic discovery. J. Ind. Microbiol. Biotechnol. 46, 257-271 (2019).

24. Jenner, M. et al. Substrate specificity in ketosynthase domains from trans-AT polyketide synthases. Angew. Chem. Int. Ed. Engl. 52, 1143-1147 (2013).

25. Irschik, $\mathrm{H}$. et al. Analysis of the sorangicin gene cluster reinforces the utility of a combined phylogenetic/retrobiosynthetic analysis for deciphering natural product assembly by trans-AT PKSs. ChemBioChem 11, 1840-1849 (2010).

26. Kampa, A. et al. Metagenomic natural product discovery in lichen provides evidence for specialized biosynthetic pathways in diverse symbioses. Proc. Natl Acad. Sci. USA 110, 3129-3127 (2013).

27. Nakabachi, A. et al. Defensive bacteriome symbiont with a drastically reduced genome. Curr. Biol. 23, 1478-1484 (2013).

28. Teta, R. et al. Genome mining reveals trans-AT polyketide synthase directed antibiotic biosynthesis in the bacterial phylum bacteroidetes. ChemBioChem 11, 2506-2512 (2010).

29. Ueoka, R. et al. Metabolic and evolutionary origin of actin-binding polyketides from diverse organisms. Nat. Chem. Biol. 11, 705-712 (2015).

30. Chevrette, M. G. et al. Evolutionary dynamics of natural product biosynthesis in bacteria. Nat. Prod. Rep. 37, 566-599 (2020).

31. Fisch, K. M. et al. Polyketide assembly lines of uncultivated sponge symbionts from structure-based gene targeting. Nat. Chem. Biol. 5, 494-501 (2009).

32. Land, T. A., Fizzano, P. \& Kodner, R. B. Measuring cluster stability in a large scale phylogenetic analysis of functional genes in metagenomes using pplacer. Trans. Comput. Biol. Bioinform. 13, 341-349 (2016).

33. Matsen, F. A., Kodner, R. B. \& Armbrust, E. V. Pplacer: linear time maximumlikelihood and Bayesian phylogenetic placement of sequences onto a fixed reference tree. BMC Bioinformatics 11, 538 (2010).

34. Piel, J. A polyketide synthase-peptide synthetase gene cluster from an uncultured bacterial symbiont of Paederus beetles. Proc. Natl Acad. Sci. USA 99, 14002-14007 (2002).

35. Wilson, M. C. et al. An environmental bacterial taxon with a large and distinct metabolic repertoire. Nature 506, 58-62 (2014).

36. Piel, J. et al. Antitumor polyketide biosynthesis by an uncultivated bacterial symbiont of the marine sponge Theonella swinhoei. Proc. Natl Acad. Sci. USA 101, 16222-16227 (2004)

37. Meoded, R. A. et al. A polyketide synthase component for oxygen insertion into polyketide backbones. Angew. Chem. Int. Ed. Engl. 57, 11644-11648 (2018).

38. Weber, T. et al. antiSMASH 3.0-a comprehensive resource for the genome mining of biosynthetic gene clusters. Nucleic Acids Res. 43, W237-W243 (2015).

39. Becerril, A. et al. Discovery of cryptic largimycins in Streptomyces reveals novel biosynthetic avenues enriching the structural diversity of the leinamycin family. ACS Chem. Biol. 15, 1541-1553 (2020).

40. Pan, G. et al. Discovery of the leinamycin family of natural products by mining actinobacterial genomes. Proc. Natl Acad. Sci. USA 114 E11131-E11140 (2017).
41. Shafi, J., Tian, H. \& Ji, M. S. Bacillus species as versatile weapons for plant pathogens: a review. Biotechnol. Biotec. Eq. 31, 446-459 (2017).

42. Genin, S. Molecular traits controlling host range and adaptation to plants in Ralstonia solanacearum. New Phytol. 187, 920-928 (2010).

43. Scherlach, K., Partida-Martinez, L. P., Dahse, H. M. \& Hertweck, C. Antimitotic rhizoxin derivatives from a cultured bacterial endosymbiont of the rice pathogenic fungus Rhizopus microsporus. J. Am. Chem. Soc. 128, 11529-11536 (2006)

44. Loper, J. E. et al. Rhizoxin analogs, orfamide A and chitinase production contribute to the toxicity of Pseudomonas protegens strain Pf-5 to Drosophila melanogaster. Environ. Microbiol. 18, 3509-3521 (2016).

45. Remenant, B. et al. Ralstonia syzygii, the blood disease bacterium and some asian $R$. solanacearum strains form a single genomic species despite divergent lifestyles. PLoS ONE 6, e24356 (2011).

46. Yin, M. et al. Cycloheximide and actiphenol production in Streptomyces sp. YIM56141 governed by single biosynthetic machinery featuring an acyltransferase-less type I polyketide synthase. Org. Lett. 16, 3072-3075 (2014).

47. Lim, S. K. et al. iso-Migrastatin, migrastatin, and dorrigocin production in Streptomyces platensis NRRL 18993 is governed by a single biosynthetic machinery featuring an acyltransferase-less type I polyketide synthase. J. Biol. Chem. 284, 29746-29756 (2009)

48. Schneider-Poetsch, T. et al. Inhibition of eukaryotic translation elongation by cycloheximide and lactimidomycin. Nat. Chem. Biol. 6, 209-217 (2010).

49. Ueoka, R. et al. Genome-based identification of a plant-associated marine bacterium as a rich natural product Source. Angew. Chem. Int. Ed. Engl. 57, 14519-14523 (2018)

50. Feil, H. et al. Comparison of the complete genome sequences of Pseudomonas syringae pv. syringae B728a and pv. tomato DC3000. Proc. Natl Acad. Sci. USA 102, 11064-11069 (2005).

51. Bertin, M. J. et al. The phormidolide biosynthetic gene cluster: a trans-AT PKS pathway encoding a toxic macrocyclic polyketide. ChemBioChem 17, 164-173 (2016).

52. Chung, E. J., Park, J. A., Jeon, C. O. \& Chung, Y. R. Gynuella sunshinyii gen. nov., sp. nov., an antifungal rhizobacterium isolated from a halophyte, Carex scabrifolia Steud. Int. J. Syst. Evol. Microbiol. 65, 1038-1043 (2015).

53. Severin, V. Ein neues pathogenes Bakterium an Hanf-Xanthomonas campestris pathovar. cannabis. Arch. Phytopathol. Plant Prot. 14, 7-15 (1978).

54. Netsu, O., Kijima, T. \& Takikawa, Y. Bacterial leaf spot of hemp caused by Xanthomonas campestris pv. cannabis in Japan. J. Gen. Plant Pathol. 80, 164-168 (2014).

55. Jacobs, J. M., Pesce, C., Lefeuvre, P. \& Koebnik, R. Comparative genomics of a cannabis pathogen reveals insight into the evolution of pathogenicity in Xanthomonas. Front. Plant Sci. 6, 431 (2015).

56. Kaida, D. et al. Spliceostatin A targets SF3b and inhibits both splicing and nuclear retention of pre-mRNA. Nat. Chem. Biol. 3, 576-583 (2007).

57. Liu, X. et al. Genomics-guided discovery of thailanstatins A, B, and C As premRNA splicing inhibitors and antiproliferative agents from Burkholderia thailandensis MSMB43. J. Nat. Prod. 76, 685-693 (2013).

58. Watrous, J. et al. Mass spectral molecular networking of living microbial colonies. Proc. Natl Acad. Sci. USA 109, E1743-E1752 (2012).

59. Kautsar, S. A., van der Hooft, J. J. J., de Ridder, D. \& Medema, M. H. BiG-SLiCE: A highly scalable tool maps the diversity of 1.2 million biosynthetic gene clusters, GigaScience, 10, giaal54 (2021).

60. Chaumeil, P. A., Mussig, A. J., Hugenholtz, P. \& Parks, D. H. GTDB-Tk: a toolkit to classify genomes with the Genome Taxonomy Database. Bioinformatics 36, 1925-1927 (2019).

61. Miller, I. J., Chevrette, M. G. \& Kwan, J. C. Interpreting microbial biosynthesis in the genomic age: Biological and practical considerations. Mar. Drugs 15, 165 (2017).

62. Miller-Wideman, M. et al. Herboxidiene, a new herbicidal substance from Streptomyces chromofuscus A7847. Taxonomy, fermentation, isolation physico-chemical and biological properties. J. Antibiot. 45, 914-921 (1992).

63. Ling, Y. et al. Pre-mRNA splicing repression triggers abiotic stress signaling in plants. Plant J. 89, 291-309 (2017).

64. Allingham, J. S., Klenchin, V. A. \& Rayment, I. Actin-targeting natural products: structures, properties and mechanisms of action. Cell Mol. Life Sci. 63, 2119-2134 (2006)

65. Mosey, R. A. \& Floreancig, P. E. Isolation, biological activity, synthesis, and medicinal chemistry of the pederin/mycalamide family of natural products. Nat. Prod. Rep. 29, 980-995 (2012).

66. Blin, K. et al. antiSMASH 4.0-improvements in chemistry prediction and gene cluster boundary identification. Nucleic Acids Res. 45, 36-41 (2017)

67. Zhang, L. H. et al. Characterization of giant modular PKSs provides insight into genetic mechanism for structural diversification of aminopolyol polyketides. Angew. Chem. Int. Ed. Engl. 56, 1740-1745 (2017). 
68. Keatinge-Clay, A. T. Polyketide synthase modules redefined. Angew. Chem. Int. Ed. Engl. 56, 4658-4660 (2017).

69. Medema, M. H. et al. Minimum Information about a Biosynthetic Gene cluster. Nat. Chem. Biol. 11, 625-631 (2015).

70. Letunic, I., Doerks, T. \& Bork, P. SMART: recent updates, new developments and status in 2015. Nucleic Acids Res. 43, D257-D260 (2015).

71. Burch, A. Y. et al. Pseudomonas syringae coordinates production of a motilityenabling surfactant with flagellar assembly. J. Bacteriol. 194, 1287-1298 (2012).

72. Innerebner, G., Knief, C. \& Vorholt, J. A. Protection of Arabidopsis thaliana against leaf-pathogenic Pseudomonas syringae by Sphingomonas strains in a controlled model system. Appl. Environ. Microbiol. 77, 3202-3210 (2011).

\section{Acknowledgements}

We thank Dr. Christine M. Vogel for her guidance with plant assays, and Satria A. Kautsar for providing a dataset of trans-AT PKS gene clusters identified in metagenomeassembled genomes. J.P. gratefully acknowledges funding by the European Research Council (ERC) under the European Union's Horizon 2020 research and innovation program (grant agreement no. 742739) and the SNF (407240_1167051, NFP 72). M.H.M. is supported by a Veni grant (863.15.002) from the Netherlands Organization for Scientific Research (NWO). M.G.C. is supported through grant 2020-67012-31772 (accession 1022881) from the USDA National Institute of Food and Agriculture and E.J.N.H gratefully acknowledges funding by LOEWE TBG.

\section{Author contributions}

E.J.N.H., R.U., M.G.C., F.H., X.L., J.P., and M.H.M. designed research, E.J.N.H., X.L., and M.H.M. compiled and analyzed the training dataset. X.L., M.G.C., and M.H.M. designed and implemented computational algorithms. E.J.N.H., M.G.C., F.H., H.M., and M.H.M. validated algorithms, E.JN.H., M.G.C., F.H., H.M., and M.H.M. analyzed results, E.J.N.H., F.H., and S.L.-M. predicted biosynthetic models, and E.J.N.H., R.U., and S.L.-M. cultured organisms, extracted and purified compounds. R.U. and S.L.-M. performed structure elucidation. S.L.-M. performed bioassays. E.J.N.H., M.G.C., F.H., J.P., and M.H.M. wrote the manuscript with contributions from all authors.

\section{Competing interests}

M.H.M. is a co-founder of Design Pharmaceuticals and a member of the scientific advisory board of Hexagon Bio. All other authors have no competing interests to declare.

\section{Additional information}

Supplementary information The online version contains supplementary material available at https://doi.org/10.1038/s41467-021-21163-x.

Correspondence and requests for materials should be addressed to J.P. or M.H.M.

Peer review information Nature Communications thanks Nigel Mouncey, Youming Zhang, and the other, anonymous reviewer(s) for their contribution to the peer review of this work.

Reprints and permission information is available at http://www.nature.com/reprints

Publisher's note Springer Nature remains neutral with regard to jurisdictional claims in published maps and institutional affiliations.

(c) (i) Open Access This article is licensed under a Creative Commons Attribution 4.0 International License, which permits use, sharing, adaptation, distribution and reproduction in any medium or format, as long as you give appropriate credit to the original author(s) and the source, provide a link to the Creative Commons license, and indicate if changes were made. The images or other third party material in this article are included in the article's Creative Commons license, unless indicated otherwise in a credit line to the material. If material is not included in the article's Creative Commons license and your intended use is not permitted by statutory regulation or exceeds the permitted use, you will need to obtain permission directly from the copyright holder. To view a copy of this license, visit http://creativecommons.org/ licenses/by/4.0/.

(C) The Author(s) 2021 